\title{
MODEL SPECTRAL ENERGY DISTRIBUTIONS OF CIRCUMSTELLAR DEBRIS DISKS. I. ANALYTIC DISK DENSITY DISTRIBUTIONS
}

\author{
Sebastian Wolf and Lynne A. Hillenbrand \\ California Institute of Technology, 1201 East California Boulevard, MS 105-24, Pasadena, CA 91125 \\ Received 2003 April 17; accepted 2003 June 20
}

\begin{abstract}
We present results of a study aimed at deriving fundamental properties of circumstellar debris disks from observed infrared-to-submillimeter spectral energy distributions (SEDs). This investigation is motivated by increasing telescope/detector sensitivity, in particular the expected availability of the Space Infrared Telescope Facility (SIRTF) followed by the Stratospheric Observatory for Infrared Astronomy (SOFIA), which will enable detailed studies with large source samples of late-stage circumstellar disk and planetary system evolution. We base our study on an analytic model of the disk density distribution and geometry, taking into account existing constraints from observations and results of theoretical investigations of debris disks. We also outline the effects of the most profound characteristics of circumstellar dust, including the grain size distribution and dust chemical composition. In particular, we find that an increasing iron content in silicates mainly causes an increase of the dust absorption efficiency and thus increases the dust reemission continuum. Furthermore, the influence of the $s p^{2} / s p^{3}$ hybridization ratio in carbon grains on the SED is examined. We investigate the influence of various parameters on the resulting dust scattering and absorption/reemission SED and discuss the possibility for distinguishing between different disks from their infrared to submillimeter spectra. The strength and shape of amorphous silicate may be particularly diagnostic of debris disk evolutionary stages. Since the appearance of these features at 10 and $20 \mu \mathrm{m}$ depends on the relative abundance of small grains and therefore the minimum grain size and slope of the grain size distribution, they can be used to trace recent collisional processes in debris disks. Thus, debris disk surveys containing statistically large numbers of objects should reveal the likelihood of collisions and therefore the evolution of dust/ planetesimals in debris disks. The results of our study underline the importance of knowledge of the stellar photospheric flux, especially in the near- to mid-infrared wavelength range, for a proper analysis of debris disk SEDs: while the quality of subtraction of the direct stellar light at far-infrared wavelengths determines the accuracy of the mass estimate in the disk, our simulations show that the remaining stellar contribution due to scattering at near- to mid-infrared wavelengths constrains the dust grain size and chemical composition, for example, the iron abundance in silicate grains.
\end{abstract}

Subject headings: circumstellar matter — methods: numerical —

planetary systems: protoplanetary disks — radiative transfer

\section{INTRODUCTION}

Debris disks are solar system-sized dust disks with micron-sized grains produced as by-products of collisions between asteroid-like bodies left over from the planet formation process. In the case of our solar system, the debris of Jupiter-family short-period comets and colliding asteroids represents the dominant source of zodiacal dust located between Mars and Jupiter. A second belt of dust is located beyond the orbit of Neptune (see, e.g., Dermott et al. 1992; Liou, Dermott, \& Xu 1995). Besides the solar system, optical to mid-infrared (MIR) images of $\beta$ Pic (see, e.g., Kalas \& Jewitt 1995; Weinberger, Becklin, \& Zuckerman 2003) and submillimeter images of Vega, Fomalhaut, and $\epsilon$ Eri (Holland et al. 1998; Greaves et al. 1998) have revealed spatially resolved debris disks that were first inferred from observations of infrared flux excesses above photospheric values with IRAS. Based on studies with the Infrared Space Observatory (ISO), the disk fraction is thought to decrease dramatically with age, amounting to much less than $10 \%$ for stars with ages $\geq 1$ Gyr (e.g., Spangler et al. 2001; see also Habing et al. 2001). Planetary debris disks are assumed to represent the almost final stage of the circumstellar disk evolution process; i.e., they are the evolutionary products of ongoing planet formation.
In contrast to optically thick young circumstellar disks around Herbig $\mathrm{Ae} / \mathrm{Be}$ and $\mathrm{T}$ Tauri stars with spatial structures dominated by gasdynamics, the much lower optical depth and lower gas-to-dust mass ratio in debris disks (Zuckermann, Forveille, \& Kastner 1995; Dent et al. 1995; Artymowicz 1997; Liseau \& Artymowicz 1998; Greaves, Coulson, \& Holland 2000a; Thi et al. 2001) let the stellar radiation, in addition to gravitation, be responsible for the disk structure. The PoyntingRobertson effect, stellar wind drag, and (if existing) gravitational stirring by embedded planets are all important in determining the dust population and disk structure (Liou \& Zook 1999; Grady et al. 2000; Moro-Martín \& Malhotra 2002). Similar to T Tauri-like disks, however, embedded planets may alter the debris disk structure remarkably (see, e.g., Gor'kavyi et al. 1997; Kenyon \& Bromley 2001). While planets may open gaps in gasdominated young circumstellar disks (see, e.g., Bryden et al. 1999, 2000; Kley 2000), they decrease the particle density within their orbits in the case of a debris disk, provided that the dust sources are located outside the planet's orbit.

Since the mass of small grains in debris disks, and therefore the thermal dust reemission from these disks, is much smaller than in the case of $\mathrm{T}$ Tauri disks, only a very limited 
sample of observations exists so far. However, because of the high sensitivity of the MIR detectors aboard the Space Infrared Telescope Facility (SIRTF), a substantial increase in the total number and in the specific information about debris disks is expected (e.g., Meyer 2001). In order to allow a quick classification of the basic characteristics of observed objects, we perform a preparatory study with the goal of revealing the influence of major disk and dust parameters, such as the inner/outer radius, density distribution, grain size distribution, composition, etc., on the emergent spectral energy distributions (SEDs) of debris disk systems.

Our first investigation, described in this publication, is based on a simply structured, optically thin analytic disk model with a limited number of basic parameters. This approach allows us to distinguish clearly between the influences of different model parameters. Furthermore, it provides an easy-to-reproduce, flux-scalable (flux is directly proportional to dust mass in the optically thin limit) database of debris disk SEDs. ${ }^{1}$ In order to consider a reasonable model setup and parameter ranges, we use boundary constraints given by previous observations and numerical simulations.

In $\S 2$ we describe the basic model and the method by which the SEDs have been calculated. In $\S \S 3,4$, and 5 we discuss specific properties of different species expected in debris disks and provide a detailed analysis of the influence of the different model parameters on the SED. Finally, we derive the SIRTF-appropriate colors $m(8 \mu \mathrm{m})-m(24 \mu \mathrm{m})$ and $m(24 \mu \mathrm{m})-m(70 \mu \mathrm{m})$ from the simulated debris disk SEDs $(\S 6)$.

\section{MODEL DESCRIPTION}

According to the expected structure of debris disks, we base our simulations on the assumption of an optically thin disk, whereby the optical depth is measured in the disk midplane as seen from the star. In this case, multiple scattering of radiation and dust heating due to dust reemission can be neglected. The only radiative processes to be considered are scattering, absorption, and reemission of stellar radiation by dust grains. The two-dimensional radiative transfer problem can then be reduced to a one-dimensional problem.

In all simulations we chose the shortest wavelength of stellar emission to measure the optical depth. This wavelength amounts to $0.2 \mu \mathrm{m}$ in practice as a result of the focus on solar-type stars and the availability of optical constants. In order to satisfy the optically thin assumption in our calculations, we apply a limit for the optical depth of $\tau_{\max }=0.05$, which translates into a maximum attenuation of stellar light by $4.8 \%$ at $0.2 \mu \mathrm{m}$. Depending on the grain size and chemical composition, the absorption efficiency decreases toward longer wavelengths but may reach a local maximum in the MIR wavelength region for particular dust chemistries (see, e.g., Figs. 3 and 5 for the wavelengthdependent absorption efficiency of $\mathrm{MgSiO}_{3}$ and crystalline olivine, respectively). However, the flux of the considered stellar radiation source is small in the MIR compared to that in the ultraviolet/optical wavelength region $\left[S_{\nu}(10 \mu \mathrm{m}) / S_{\nu}(0.55 \mu \mathrm{m}) \approx 1.3 \times 10^{-4}\right.$ for the solar $\mathrm{SED}$; see below]. As a result of the small amount of dust in the

\footnotetext{
${ }^{1}$ A database with all dust SEDs presented in this publication is available at http://mc.caltech.edu/ swolf/downloads/debris.
}

disk/shell for $\tau_{\max }=0.05$, the flux reemitted in the MIR wavelength range by hot dust is also negligible for further disk heating.

Each dust grain is heated by direct stellar radiation only. Thus, the dust grain temperature is a function of the optical parameters of the grains, the incident stellar radiation, and the distance $d$ from the star. In this case the radiative transfer equation has a simple solution that allows one to derive the distance from the star at which the dust has a certain temperature. Let

$$
L_{\lambda, s}=4 \pi R_{s}^{2} \pi B_{\lambda}\left(T_{s}\right)
$$

be the monochromatic luminosity of the star (radius $R_{S}$, effective temperature $T_{s}$ ) at wavelength $\lambda$ and

$$
L_{\lambda, g}^{\mathrm{abs}}=L_{s} Q_{\lambda}^{\mathrm{abs}} \frac{\pi a^{2}}{4 \pi d^{2}}
$$

and

$$
L_{\lambda, g}^{\mathrm{emi}}=4 \pi a^{2} Q_{\lambda}^{\mathrm{abs}} \pi B_{\lambda}\left(T_{g}\right)
$$

be the absorbed and reemitted luminosity, respectively, of a dust grain with radius $a$ and resulting temperature $T_{g}$ at the (unknown) distance $d$ from the star. Using the constraint of energy conservation

$$
\int_{0}^{\infty} L_{\lambda, g}^{\mathrm{emi}} d \lambda=\int_{0}^{\infty} L_{\lambda, g}^{\mathrm{abs}} d \lambda
$$

one derives the distance of the grain from the star as

$$
d\left(T_{g}\right)=\frac{R_{s}}{2}\left[\frac{\int_{0}^{\infty} d \lambda Q_{\lambda}^{\mathrm{abs}}(a) B_{\lambda}\left(T_{s}\right)}{\int_{0}^{\infty} d \lambda Q_{\lambda}^{\mathrm{abs}}(a) B_{\lambda}\left(T_{g}\right)}\right]^{1 / 2} .
$$

If the dust sublimation temperature is known, equation (4) also allows one to estimate the sublimation radius for each dust component in the shell (characterized by the grain radius and chemical composition). The flux of the light scattered by a single dust grain amounts to

$$
L_{\lambda, g}^{\mathrm{sca}}=L_{\lambda, s} A Q_{\lambda}^{\mathrm{sca}}\left(\frac{a}{2 d}\right)^{2}
$$

where $A$ is the dust grain's albedo.

We calculate the temperature distribution of the dust based on 500 logarithmically equidistantly distributed wavelengths in the interval $[0.2 \mu \mathrm{m}, 500 \mu \mathrm{m}]$. According to equation (5), distances $d(T)$ corresponding to 500 temperatures equidistantly distributed between $2.73 \mathrm{~K}$ and the dust grain sublimation temperature of the grains are determined. The dust reemission and scattering are calculated at $200 \mathrm{log}$ arithmically equidistantly distributed wavelengths in the interval $[2 \mu \mathrm{m}, 200 \mu \mathrm{m}]$. The latter interval was chosen according to the wavelength range covered by broadband filters aboard SIRTF: 3.6-160 $\mu \mathrm{m}$. The net SED results from a simple summation of the reemitted and scattered-light contributions from all grains.

Since the SED depends on the radial density distribution only, we consider a spherical shell instead of a circumstellar disk. This simplification has no effect on the resulting temperature distribution and/or SED but reduces the number of free parameters. If a disk with an opening angle from the midplane $\theta$ but the same radial density distribution is considered instead, the SEDs shown in the subsequent sections 
have to be multiplied by the factor

$$
f_{\theta}=\frac{1}{2}\left[\cos \left(\frac{\pi-\theta}{2}\right)-\cos \left(\frac{\pi+\theta}{2}\right)\right],
$$

but the relative shape of the dust contribution to the net SED by scattering, absorption, and reemission remains unchanged. For $\theta=10^{\circ}, f_{\theta} \approx 0.087$, while for $\theta=45^{\circ}$, $f_{\theta} \approx 0.38$.

Our parameters are as follows, unless specified otherwise in subsequent sections. The applied radial density profile in the disk/shell follows a power law

$$
n(r) \propto r^{-q} .
$$

Except for a parameter study in $\S 4.1$, we use $q=1$, representing a debris disk with no perturbations by embedded planets and no particular assumptions about dust production processes (see $\S 4.1$ for a more detailed description). If not otherwise noted, the mass of the disk amounts to $10^{-10}$ $M_{\odot}$, the inner disk radius is equivalent to the dust sublimation radius, and the outer radius amounts to $100 \mathrm{AU}$. The central star has a solar-type SED (we use the solar SED published by Labs \& Neckel 1968, extended by a blackbody SED beyond $151 \mu \mathrm{m})$. The distance to the system is assumed to be $50 \mathrm{pc}$.

\section{DUST GRAIN PARAMETERS}

The dust found in young, massive circumstellar disks around $\mathrm{T}$ Tauri and Herbig Ae/Be stars consists mainly of two distinct species: a silicate component and a carbonaceous component (Savage \& Mathis 1979; Draine \& Lee 1984; Malfait et al. 1999). Based on the investigation of the interstellar extinction, Weingartner \& Draine (2001; see also Draine \& Lee 1984) derived optical properties and relative abundances of both. Despite a different size distribution of the grains in the circumstellar versus the interstellar environment (see, e.g., Mathis, Rumpl, \& Nordsieck 1977; Beckwith \& Sargent 1991; Miyake \& Nakagawa 1993), the chemical composition turned out to be very similar, allowing detailed studies of the circumstellar environment and disks of young stellar objects. However, the very complex, (magneto-)hydrodynamically dominated optically thick disks permit derivation of only very basic further information about the dust, such as the grain size distribution exponent and minimum and approximate maximum upper grain size (e.g., Wolf, Padgett, \& Stapelfeldt 2003 and references therein). In contrast to the situation for young optically thick disks, older optically thin debris disks are expected to allow a more detailed investigation of dust grain parameters, in particular the chemical components and the grain sizes. An important caveat to this claim of simpler modeling for debris disks is that because the dust dynamics are determined by a combination of radiation pressure, the Poynting-Robertson effect, and the (possible) production of dust due to collision processes and gravitational interaction with possibly embedded planets, the disk density distribution may be dictated by several additional parameters, the influence of which on the dust reemission/scattered-light SED will be discussed in detail in $\S 4$ and explored more thoroughly in a forthcoming paper.

Fortunately, existing laboratory measurements provide a rich database of dust properties expected in different environments (defined by the dust formation conditions, tem- perature, abundances of particular elements, etc.). A compilation of a large number of measured optical dust parameters has been published by Henning et al. (1999). Before applying selected chemical compositions in our investigation of the influence of disk model parameters on the observable SED, we outline in brief the most characteristic differences between the dust compositions expected to dominate.

We consider the following chemical components: (1) amorphous silicates with varying iron content from $\mathrm{Fe}$-poor through Fe-rich, (2) crystalline silicates (in particular olivine), and (3) amorphous carbon species with different graphite/diamond structure ratios. ISO spectroscopy of Herbig $\mathrm{Ae} / \mathrm{Be}$ stars revealed several further abundant chemical components, such as Fe (broad, weak emission feature at $\approx 2-4 \mu \mathrm{m}$; see Henning \& Stognienko 1996), FeO (broad emission features at $\approx 21-25 \mu \mathrm{m}$; see Henning et al. 1995), and/or FeS (see Henning \& Stognienko 1996) and $\mathrm{H}_{2} \mathrm{O}$ ice (broad emission features between $\approx 40$ and $80 \mu \mathrm{m}$; Warren 1984). For model SEDs taking into account these additional components see, e.g., Malfait et al. (1999), Bouwman et al. (2000), and Meeus et al. (2001). However, the SEDs of debris disks measured so far do not allow one to perform a comparably detailed chemical analysis; SIRTF is expected to be revolutionary in this regard.

We consider the dust grains to be homogeneous spheres. Although dust grains are expected to have a fractal structure, the scattering behavior is similar to that of spheres (see Lumme \& Rahola 1994 for porous dust particle light scattering). Furthermore, dust grains are expected to have a nonspherical shape (see, e.g., Elvius \& Hall 1967; Scarrott, Draper, \& Warren-Smith 1989; Hajjar \& Bastien 1996; Kastner \& Weintraub 1996; Dollfus \& Suchail 1987; Johnson \& Jones 1991; Chrysostomou et al. 2000). However, we do not consider the grains to be aligned on a large scale by magnetic fields; thus, the assumption of spherical grains is a valid approximation (Wolf, Voshchinnikov, \& Henning 2002). The optical parameters of the grains required for the estimation of the dust absorption, reemission, and scattering are derived from the complex refractive index $m=n+i k$ and the grain radius. We use measurements of the complex refractive index $m$ published by Dorschner et al. (1995; silicates) and Jäger et al. (1998; carbon). ${ }^{2}$ Furthermore, we compare selected dust properties with the optical properties of " astronomical silicate" and graphite provided by Weingartner \& Draine (2001; see also Draine \& Lee 1984) since these data are used in a large variety of simulations of circumstellar dust configurations. According to equations (5) and (6), the absorption and scattering efficiencies determine the particular contribution of each chemical component to the net SED. We consider absorption/reemission and scattering for individual grains of given composition and size over the wavelength range $0.2-200 \mu \mathrm{m}$.

The interaction of the stellar radiation field with the dust grains is described by Mie scattering theory. We calculate the Mie scattering function using the numerical solution for the estimation of the Mie scattering coefficients published by Voshchinnikov (2003), which achieves accurate results in both the small and the (arbitrarily) large size parameter regime. $^{3}$

\footnotetext{
2 The complex refractive indices are available at http://www.astro.uni-jena.de/Laboratory/Database/odata.html.

${ }^{3}$ The code for calculation of the Mie scattering coefficients is available at http://mc.caltech.edu/ swolf/miex-web/miex.htm (or contact the authors).
} 
In the following we briefly describe the silicate and carbon species considered:

Silicates.-Previous studies concerning the MIR SED of evolved stars and the $\beta$ Pic circumstellar disk revealed that a large variety of silicate species are present in these different environments. For example, Pantin, Lagage, \& Artymowicz (1997) find amorphous olivine $\left(\mathrm{MgFeSiO}_{4}\right)$ to be the dominant chemical dust component in the $\beta$ Pic disk $(55 \%$ amorphous olivine, $35 \%$ amorphous pyroxene, and $10 \%$ crystalline olivine). In contrast to this, Molster, Waters, \& Tielens (2002a, 2002b) find narrowband emission features in the SED of dust around evolved stars, clearly pointing to the existence of crystalline silicates. In order to cover this large range of different silicates, we investigate the main differences between a number of different amorphous pyroxene species $\left(\mathrm{Mg}_{x} \mathrm{Fe}_{1-x} \mathrm{SiO}_{3}\right.$ with $x=0.4,0.5,0.6$, $0.7,0.8,0.95$, and 1.0) and amorphous olivine species $\left(\mathrm{Mg}_{2 y} \mathrm{Fe}_{2-2 y} \mathrm{SiO}_{4}\right.$ with $y=0.4$ and 0.5$)$. The efficiency factors for $1 \mu \mathrm{m}$ grains are shown in Figure 1. Despite the characteristic 10 and $19 \mu \mathrm{m}$ maxima of the absorption efficiency, the most significant property is the strong dependence of the UV-to-NIR absorption efficiency on the Fe content (see Jäger et al. 1994 for a detailed compilation
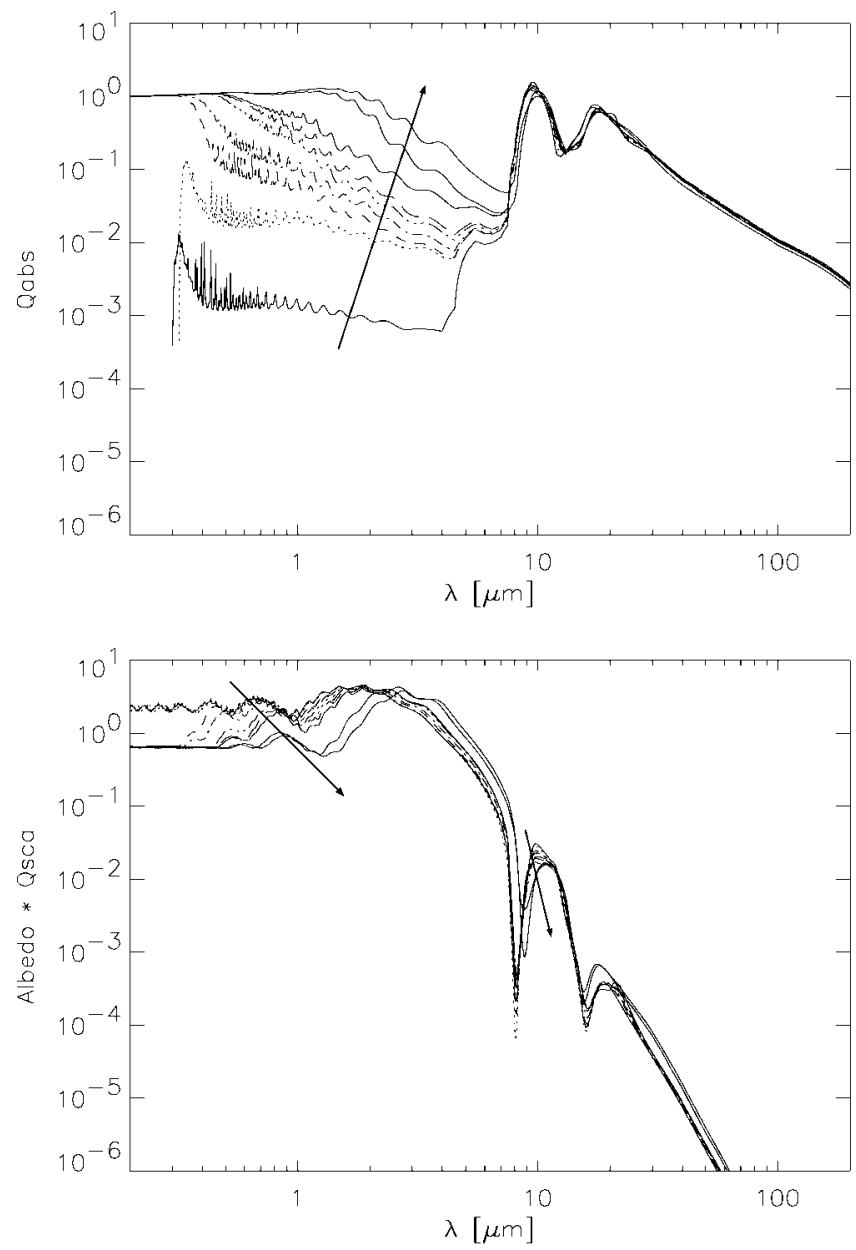

FIG. 1.-Absorption and scattering coefficients over the transition from $\mathrm{Fe}$-deficient to $\mathrm{Fe}$-rich silicates. The arrow indicates increasing iron content from $\mathrm{MgSiO}_{3} \rightarrow \mathrm{Mg}_{0.95} \mathrm{Fe}_{0.05} \mathrm{SiO}_{3} \rightarrow \mathrm{Mg}_{0.8} \mathrm{Fe}_{0.2} \mathrm{SiO}_{3} \rightarrow \mathrm{Mg}_{0.7} \mathrm{Fe}_{0.3} \mathrm{SiO}_{3} \rightarrow$ $\mathrm{Mg}_{0.6} \mathrm{Fe}_{0.4} \mathrm{SiO}_{3} \rightarrow \mathrm{Mg}_{0.5} \mathrm{Fe}_{0.5} \mathrm{SiO}_{3} \rightarrow \mathrm{Mg}_{0 .} \mathrm{Fe}_{0.6} \mathrm{SiO}_{3} \rightarrow \mathrm{MgFeSiO}_{4} \rightarrow$ $\mathrm{Mg}_{0.8} \mathrm{Fe}_{1.2} \mathrm{SiO}_{4}$. The optical parameters have been calculated for grains with a radius of $1 \mu \mathrm{m}$ and discussion of absorption bands of amorphous pyroxene). With increasing relative Fe content the absorption efficiency increases by about 3 orders of magnitude in the case of a micron-sized grain in this wavelength range from $\mathrm{Fe}$ deficient $\mathrm{MgSiO}_{3}$ to Fe-rich $\mathrm{Mg}_{0.8} \mathrm{Fe}_{1.2} \mathrm{SiO}_{4}$. Thus, the temperature and reemitted flux of the grains at a given radial distance from the star increase. These effects are
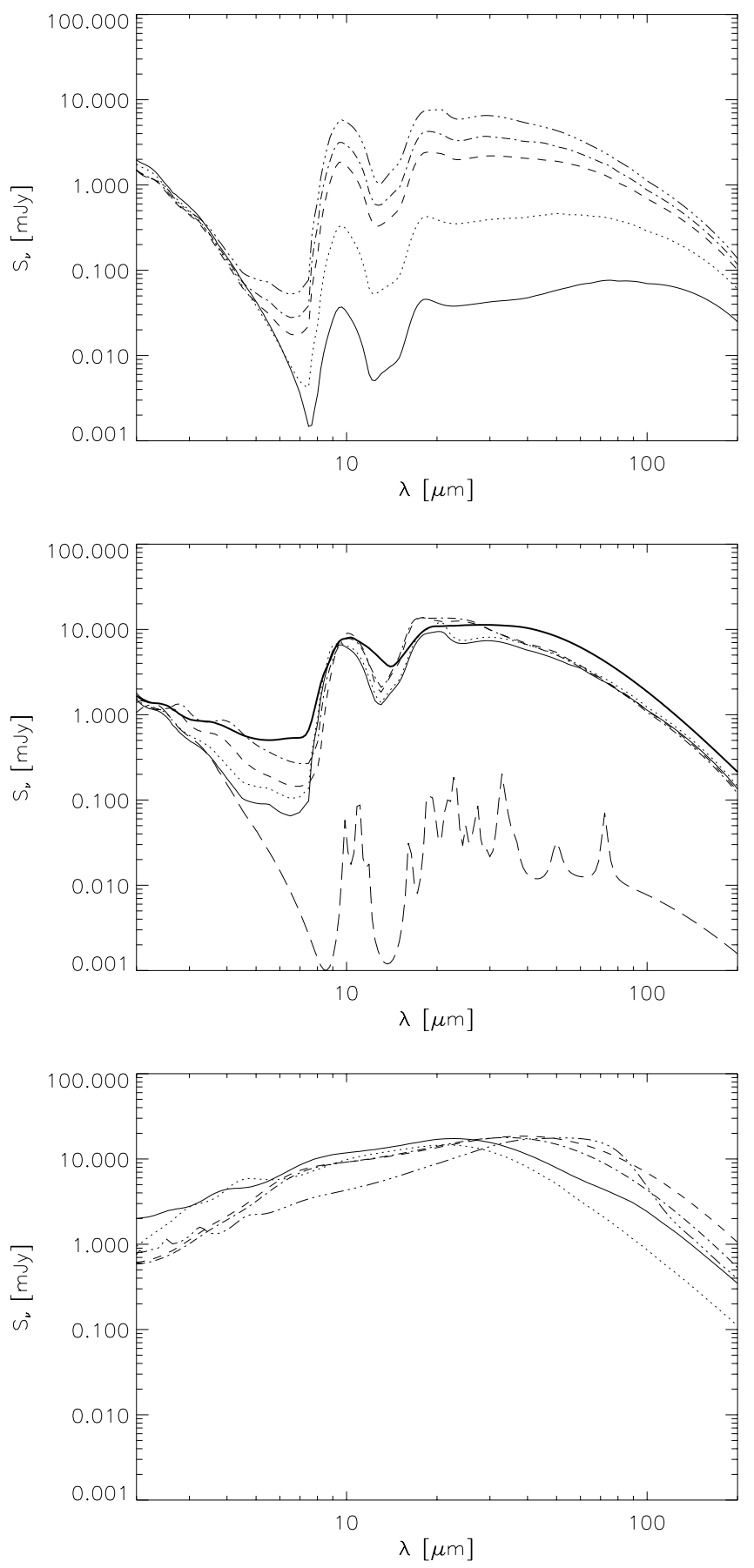

Fig. 2.-Contributions from single dust compositions. Top: $\mathrm{MgSiO}_{3}$ (solid line), $\mathrm{Mg}_{0.95} \mathrm{Fe}_{0.05} \mathrm{SiO}_{3}$ (dotted line), $\mathrm{Mg}_{0 .} \mathrm{Fe}_{0 .} \mathrm{SiO}_{3}$ (dashed line), $\mathrm{Mg}_{0.7} \mathrm{Fe}_{0.3} \mathrm{SiO}_{3}$ (dot-dashed line), $\mathrm{Mg}_{0.6} \mathrm{Fe}_{0.4} \mathrm{SiO}_{3}$ (triple-dot-dashed line). Middle: $\mathrm{Mg}_{0.5} \mathrm{Fe}_{0.5} \mathrm{SiO}_{3}$ (solid line), $\mathrm{Mg}_{0.4} \mathrm{Fe}_{0.6} \mathrm{SiO}_{3}$ (dotted line), $\mathrm{MgFeSiO}_{4}$ (dashed line), $\mathrm{Mg}_{0.8} \mathrm{Fe}_{1.2} \mathrm{SiO}_{4}$ (dot-dashed line), "astronomical silicate" (thick solid line), crystalline olivine (long-dashed line). Bottom: C at 400 (solid line), 600 (dotted line), 800 (dashed line), and $1000 \mathrm{~K}$ (dot-dashed line) and graphite (thick solid line). In all cases a single grain radius of $a=1 \mu \mathrm{m}$ and a disk mass of $10^{-10} M_{\odot}$ are assumed. 

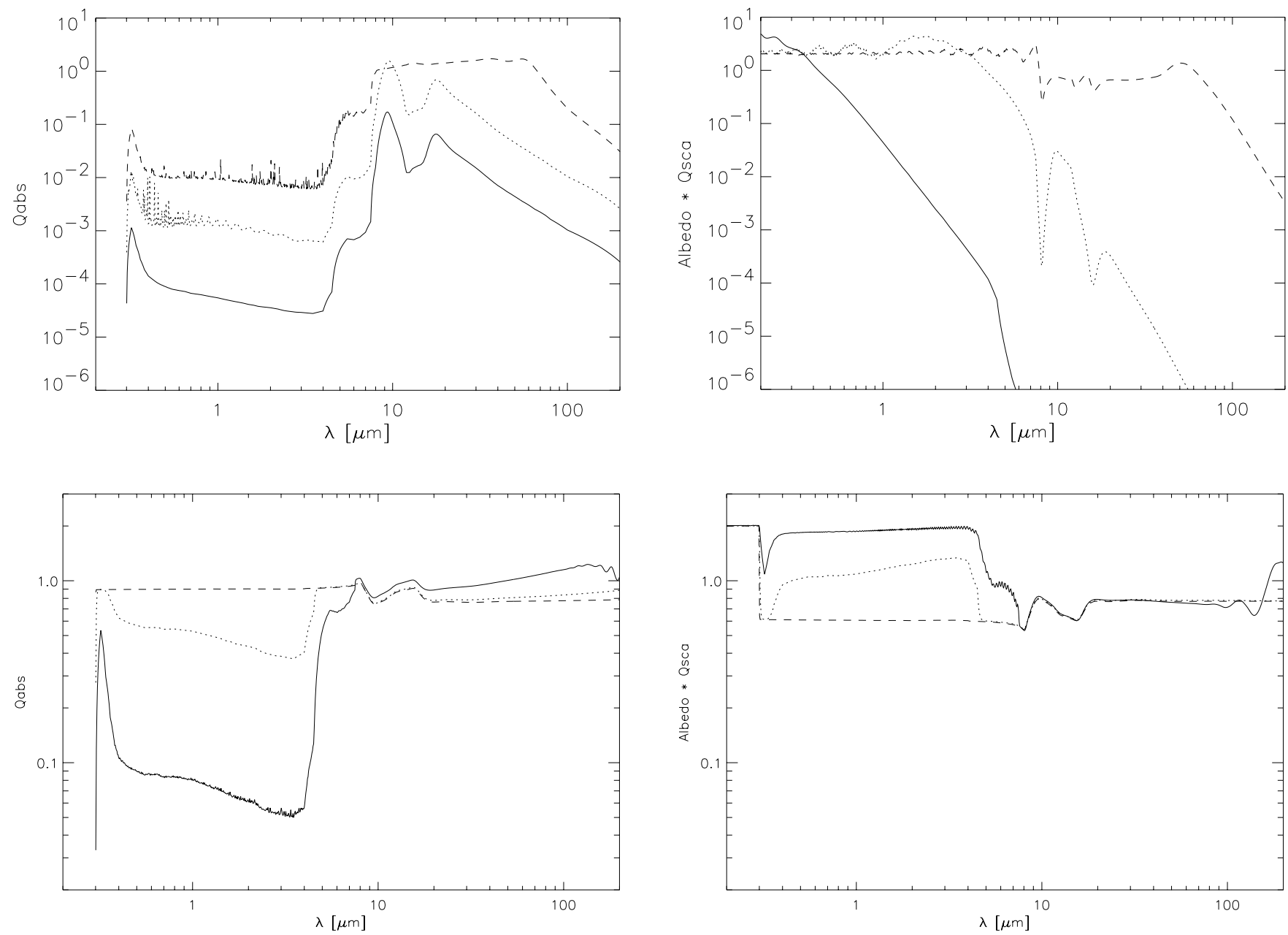

Fig. 3.-Absorption and scattering coefficients for Mg-rich/Fe-deficient silicate: $\mathrm{MgSiO}_{3}$. Top: Grain radii 0.1 (solid line), 1 (dotted line), and $10 \mu \mathrm{m}$ (dashed line). Bottom: Grain radii 0.1 (solid line), 1 (dotted line), and $10 \mathrm{~mm}$ (dashed line).

demonstrated in Figure 2, where the resulting density distribution for our standard disk model (see below) is shown. Furthermore, the comparison with astronomical silicate (Weingartner \& Draine 2001) shows that it is much more similar to Fe-rich than to Fe-poor silicates. For comparison, the scattering/reemission SED of crystalline olivine $\left(\mathrm{MgFeSiO}_{4}\right)$ is shown. Similar to Fe-poor silicates, crystalline olivine has a very low absorption efficiency in the wavelength range of stellar emission (see Fig. 5) and can therefore only be traced (by its pronounced numerous narrow emission features) either based on highly accurate photometric measurements or if a high relative amount of this material is present in the disk. Furthermore, the dependence of the efficieny factors on the grain size is illustrated in Figures 3, 4, and 5 for Fe-deficient and Fe-rich amorphous silicate and crystalline olivine.

Carbon.- It is commonly assumed that the carbonaceous dust component occurs in the form of graphite (see, e.g., Draine \& Lee 1984; Li \& Greenberg 1997; Weingartner \& Draine 2001). In contrast to this common assumption, the dust emissivity described by a power law with a spectral index of $\approx 1$ (Campbell et al. 1976; Sopka et al. 1985; Martin \& Rogers 1987; Gürtler, Kömpe, \& Henning 1996) can be explained by the occurrence of very disordered twodimensional material like amorphous carbon (Kittel 1963). Jäger, Mutschke, \& Henning (1998) synthesized structurally different carbon material with an increasing $s p^{2} / s p^{3}$ ratio by pyrolizing cellulose materials at $400^{\circ} \mathrm{C}-1000^{\circ} \mathrm{C}$. The different carbon structures therefore represent different graphite ( $s p^{2}$ hybridization) to diamond structure ( $s p^{3}$ hybridization) abundance ratios. In Figures 6 and 7 we show the efficiency factors calculated for the two extreme cases: the $400 \mathrm{~K}$ carbon modification (graphite-poor) and $1000 \mathrm{~K}$ carbon modification (graphite-rich). As these figures show, carbon has a high absorption efficiency in the UV/visual wavelength range but, in contrast to silicate species, no prominent absorption features (see also Fig. 2). Thus, it mainly adds a strong continuum to the SED. The absorption efficiency in the UV/visual wavelength range is comparable to Fe-rich silicates. With increasing sublimation temperature the range of almost constant, high absorption efficiency is extended toward longer wavelengths. The particular turnover point beyond which the absorption drops continuously increases as the grain size is increased. It is noteworthy that the absorption efficiency of graphite as published by Weingartner \& Draine (2001) is smaller than that of the other carbon configurations in the MIR wavelength range.

Finally, we illustrate the importance of scattering of stellar radiation by the dust in the case of iron-deficient and iron-rich silicate (see Fig. 8). Mainly determined by the solar-type SED of the star, the contribution of scattering to 

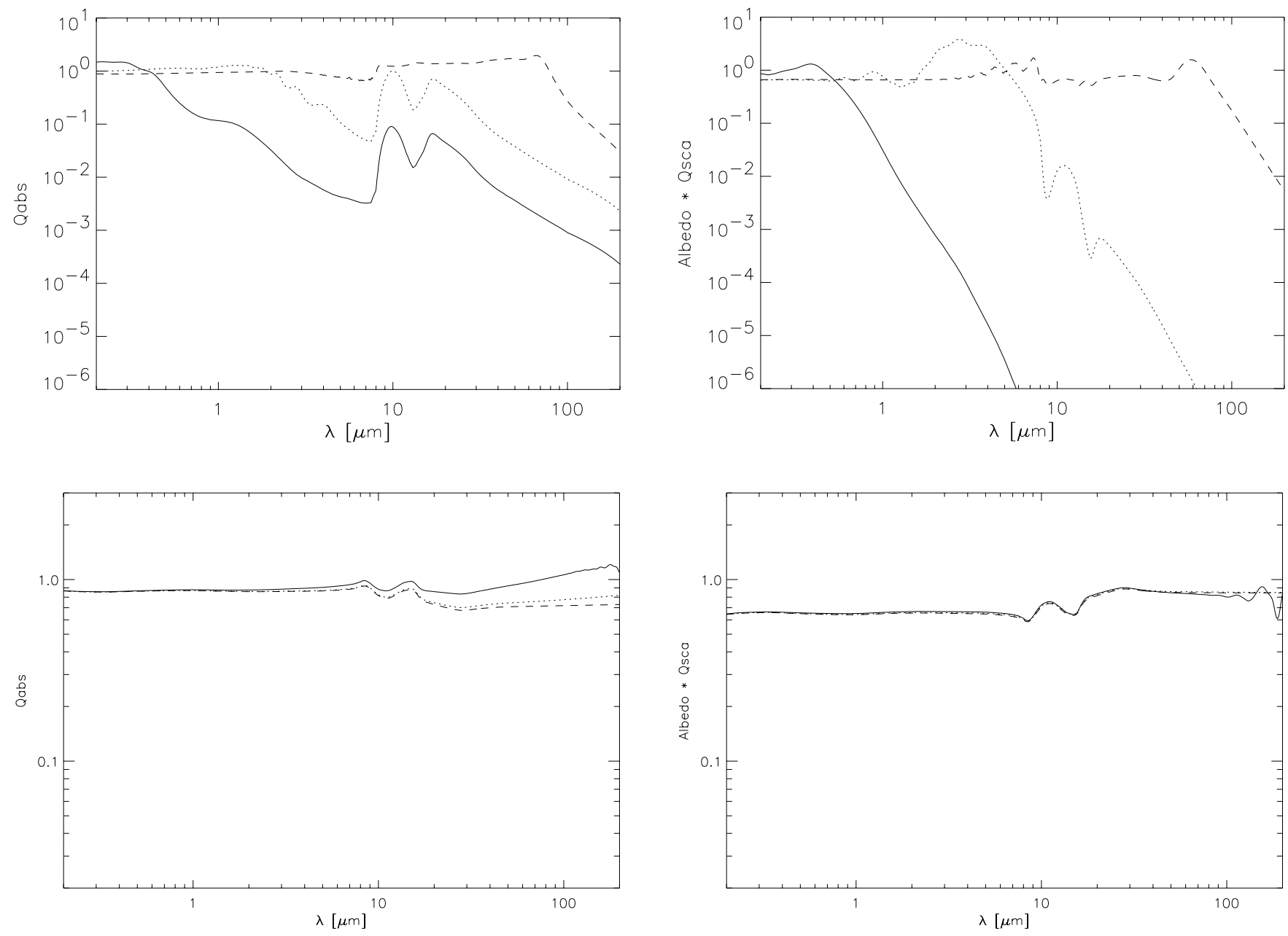

FIG. 4.-Absorption and scattering coefficients for Mg-poor/Fe-rich silicate: $\mathrm{Mg}_{0.8} \mathrm{Fe}_{1.2} \mathrm{SiO}_{4}$. Lines are the same as in Fig. 3 .

the resulting SED is negligible beyond $\approx 10 \mu \mathrm{m}$. At shorter wavelengths, however, the lower absorption efficiency in the case of an iron-deficient silicate results in a larger relative contribution of the scattered light, while the iron-rich amorphous olivine dust reemission dominates at wavelengths of a few microns. Thus, if iron-poor silicates or crystalline olivine are the dominating dust species, spectral features of the star will be present in the near-infrared (NIR) to MIR wavelength range even after correct subtraction of the stellar SED (e.g., resulting from independent stellar models).

\section{PARAMETER STUDY}

In this section we explore the parameter space allowed by our adopted analytic disk model. We are strongly guided by observations and theoretical constraints in considering viable values for the disk density distribution, inner and outer disk radius, and grain size distribution.

\subsection{Disk Density Distribution}

Based on hydrostatic models, the radial density distribution in T Tauri disks is proposed to be in the range

$$
n(r) \propto r^{-(1.9-2.4)}
$$

(see, e.g., Kenyon \& Hartmann 1987; D’Alessio et al. 1999; Chiang \& Goldreich 1999). Detailed modeling of the NIR- to-millimeter appearance of several spatially resolved $\mathrm{T}$ Tauri disks has confirmed these predictions (see, e.g., Burrows et al. 1996; Stapelfeldt et al. 1998; Cotera et al. 2001; Wood et al. 2002; Wolf et al. 2003). Optically thin debris disks, which, in contrast to $\mathrm{T}$ Tauri disks, are not dominated by gas, are assumed to cover this range of radial density profiles as well but also to manifest values outside of this range.

In the most simple model of debris disks, structure is dominated exclusively by gravitation, radiation pressure, and Poynting-Robertson drag. In a collisionless system without sources or sinks and grains in circular orbits, the exponent $q$ amounts to 1 (see, e.g., Briggs 1962). This "classical solution" approximately represents the overall distribution of dust in the solar system (see, e.g., Ishimoto 2000). However, as, for instance, Gor'kavyi et al. (1997) pointed out, there exist several effects that may change this distribution remarkably, including resonance effects with planets, gravitational encounters with planets that occur in the form of elastic gravitational scattering, mutual collisions of particles, evaporation of dust grains, and existence of sources of dust with highly eccentric orbits (such as Encke's comet in the case of the solar system; see, e.g., Whipple 1976; Sykes 1988; Epifani et al. 2001). The resulting density distribution is in most cases no longer described by a single power law as stated in equation (8) but depends strongly on the properties 

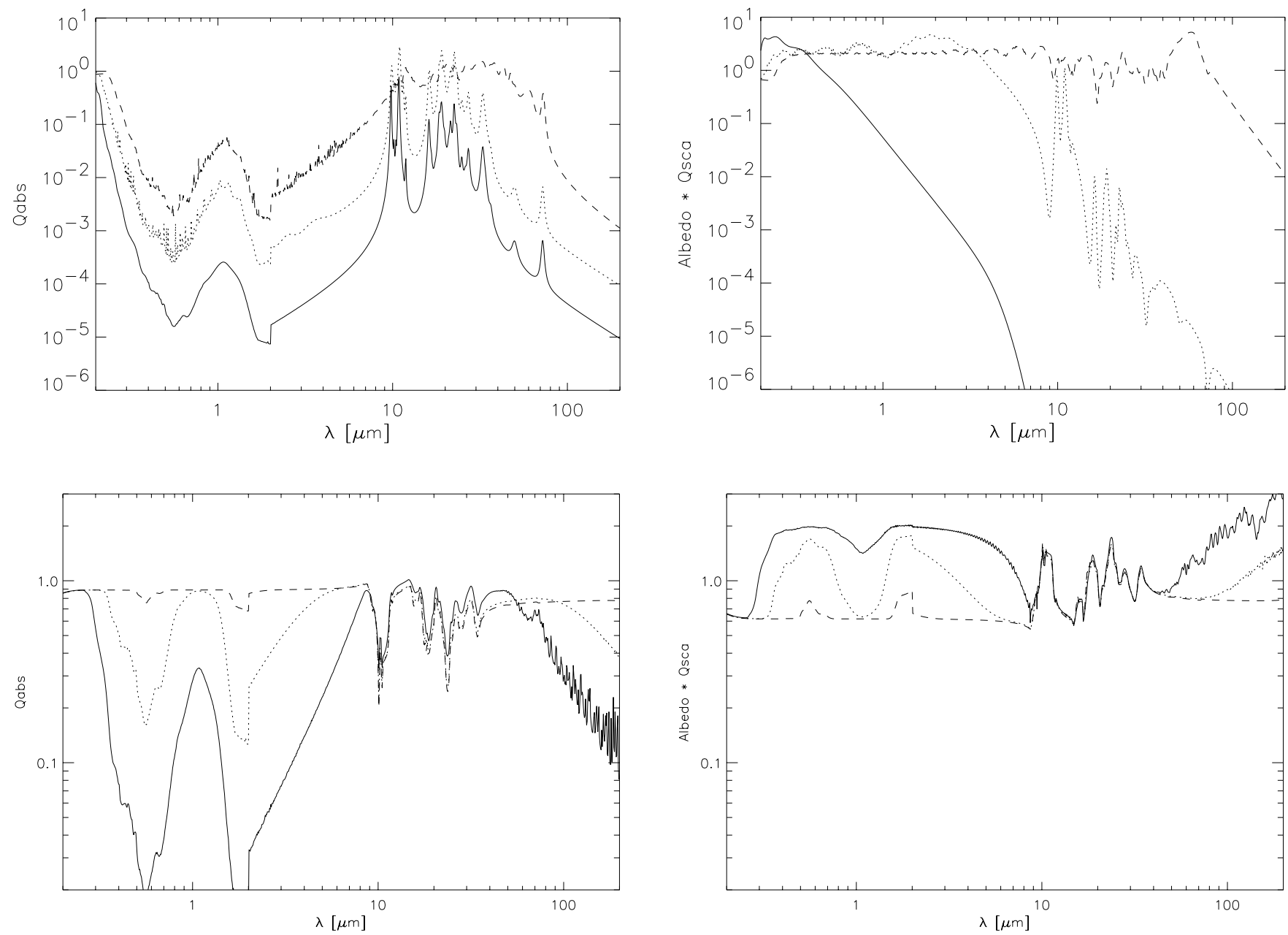

FIG. 5.-Absorption and scattering coefficients for olivine (crystalline, but not oriented): $\mathrm{Mg}_{1.9} \mathrm{Fe}_{0.1} \mathrm{SiO}_{4}$. Lines are the same as in Fig. 3 .

(mainly the orbits and masses of planets and dust sources) of each particular disk/planetary system. The exponents of the radial density distribution derived for different radial zones in the model of the solar system reach from $q=1.0$ to 2.4 (Gor'kavyi et al. 1997). Another simply structured debris disk would be a cloud of grains moving outward from the center in hyperbolic trajectories. This solution, which applies to so-called $\beta$-meteorites in the interplanetary space, is described by $q=2$ (see, e.g., Lecalvier des Etangs, VidalMadjar, \& Ferlet 1998; Ishimoto \& Mann 1999). In the case of $\beta$ Pic, visible observations of the scattered starlight and MIR images show that the outer disk (at radii greater than $100 \mathrm{AU})$ can be described by a power law in the range [1.5, 2.3] (Artymowicz, Burrows, \& Paresce 1989; Kalas \& Jewitt 1995; Smith \& Terrile 1984; Pantin et al. 1997). ${ }^{4}$ In the following we consider the cases $q=1.0,1.5,2.0$, and 2.5.

We find that for a given grain size and chemistry, the radial density profile, described by the exponent $q$ (see eq. [8]), determines the overall slope of the SED (see Fig.

${ }^{4}$ We want to remark that Trilling, Brown, \& Rivkin (2000; see also Trilling \& Brown 1998) reported the detection of circumstellar disks around $55 \mathrm{Cnr}, \rho \mathrm{CrB}$, and HD 210277, which are planet-harboring systems (Butler et al. 1997; Noyes et al. 1997; Marcy et al. 1999). From scattered-light images these authors derive a volume density profile described by $q=4$. However, since these measurements could not be confirmed so far by later, independent (see, e.g., Jayawardhana et al. 2002) measurements, we do not take such steep density profiles into account.
9). The relative strength of the spectral features is not affected by $q$. The steeper the density profile, i.e., the larger the exponent $q$, the larger the relative amount of dust located in the inner regions of the disk. Since the dust temperature increases toward the star, this results in an increase of the NIR/MIR-to-(sub)millimeter luminosity ratio. However, the net reemission of the disk also increases with increasing steepness of the density profile since the relative number of hotter and therefore more luminous grains increases.

\subsection{Inner Gaps in the Disk}

Observed SEDs of selected disks around T Tauri and debris-type disks have been found to show hints of inner cavities such that the inner radius of the disk is apparently much larger than the sublimation radius of possible dust species. Examples among T Tauri disks are GM Aurigae (Koerner, Sarget, \& Beckwith 1993; Rice et al. 2003) and TW Hya (Calvet et al. 2002). In several prominent debris disks, inner cavities have been found as well: $\beta$ Pic (inner radius of $20 \mathrm{AU}^{5}$ ), HR 4796A (30-50 AU), $\epsilon$ Eri (50 AU), Vega (80 AU), and Fomalhaut (125 AU) (see, e.g., Dent

${ }^{5}$ We want to remark that this value derived by Dent et al. (2000) from optical-to-submillimeter spectroscopy is in contrast to the result given by Weinberger et al. (2003), who assume a substantial amount of dust grains within that radius based on spatially resolved MIR spectroscopy (see $\S 4.4$ ). 

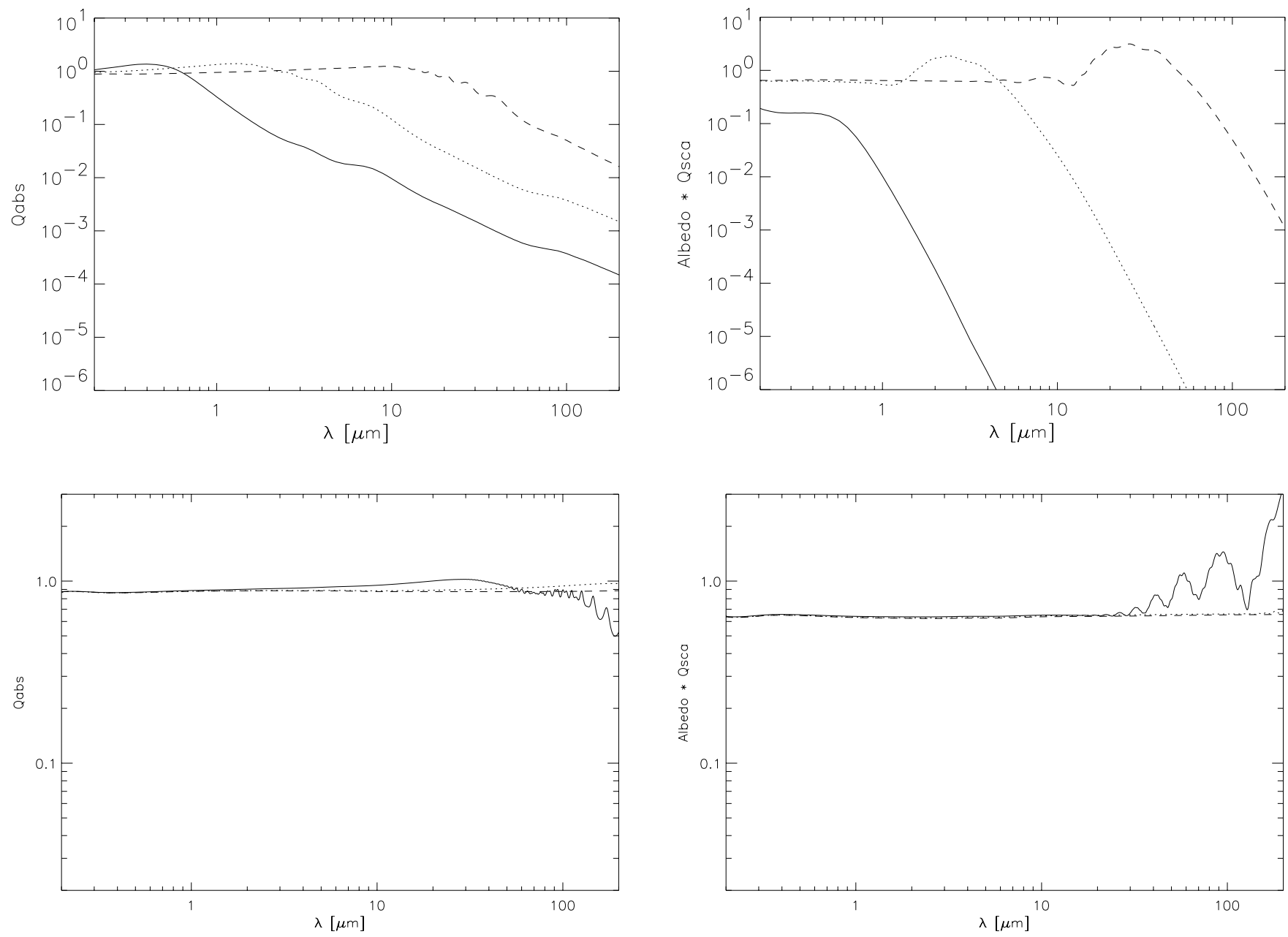

FIG. 6.-Absorption and scattering coefficients for carbon: $400 \mathrm{~K}$ configuration ("graphite poor"). Lines are the same as in Fig. 3.

et al. 2000; Greaves, Mannings, \& Holland 2000b; Wilner et al. 2002; Holland et al. 2003). Taking into account the physical processes responsible for the spatial dust density distribution in debris disks, scattering of particles by massive planets is assumed to be the major effect in explaining these large inner holes: dust grains drifting inward as a result of the Poynting-Robertson effect are likely to be scattered into larger orbits resulting in a lower dust number density within the planet's orbit. Based on the assumed cavity sizes of the debris disks quoted above (relative to the total sizes of these disks), we consider the cases $0.1,1.0$, and $10 \mathrm{AU}$ and compare the resulting SED to the case of a disk without any gap, that is, extending inward to the dust sublimation radius.

Clearing the inner disk results in a loss of warm dust that is mainly responsible for the NIR/MIR shape of the SED. With increasing gap size the minimum value of the MIR flux becomes smaller and is shifted toward longer wavelengths (see Fig. 10). Because of the higher temperature and therefore more significant contribution to the NIR/MIR spectrum by small grains, the decrease of the flux in this wavelength range is more pronounced when the relative number of small grains in the system goes up. Keeping the disk mass constant, the flux in the millimeter region (not accessible by SIRTF) increases slightly, but the net flux is smaller compared to a disk without an inner gap. This is because the fraction of the stellar flux absorbed by a single dust grain decreases with increasing radial distance from the star (see eq. [2]). Applying the same explanation to the scattering cross section (see eq. [6]), the slight decrease of the scattered stellar light with increasing gap size can be explained by analogy.

\subsection{Outer Disk Radius}

Similar to T Tauri disks, circumstellar disks at the late stage of their evolution appear to show a large range of possible radii, typically in the range of about 100 to several hundred AU. So far, scattered-light images, submillimeter maps, and SEDs revealed radii of 80 ( $\epsilon$ Eri), 120 (Vega), 125 (HR 4796), 185 (Fomalhaut), and $1000 \mathrm{AU}$ (the disk around $\beta$ Pic; see Vidal-Madjar, Lecavelier des Etangs, \& Ferlet 1998; Dent et al. 2000; Greaves et al. 2000b; Holland et al. 2003). However, scattered-light images, as well as NIR/ MIR SEDs of debris disks, are mainly determined by the pronounced spectral features and the total flux in the small grain component in the disk (see $\S 4.1$, Fig. 12), while larger grains with radii of several tens of microns and larger can be more efficiently found by (sub)millimeter measurements. One has to take into account that the abundance and spatial distribution of the small grain component, especially the one with $\beta \gtrsim 1$ (see eq. [11]), depends on the location of dust 

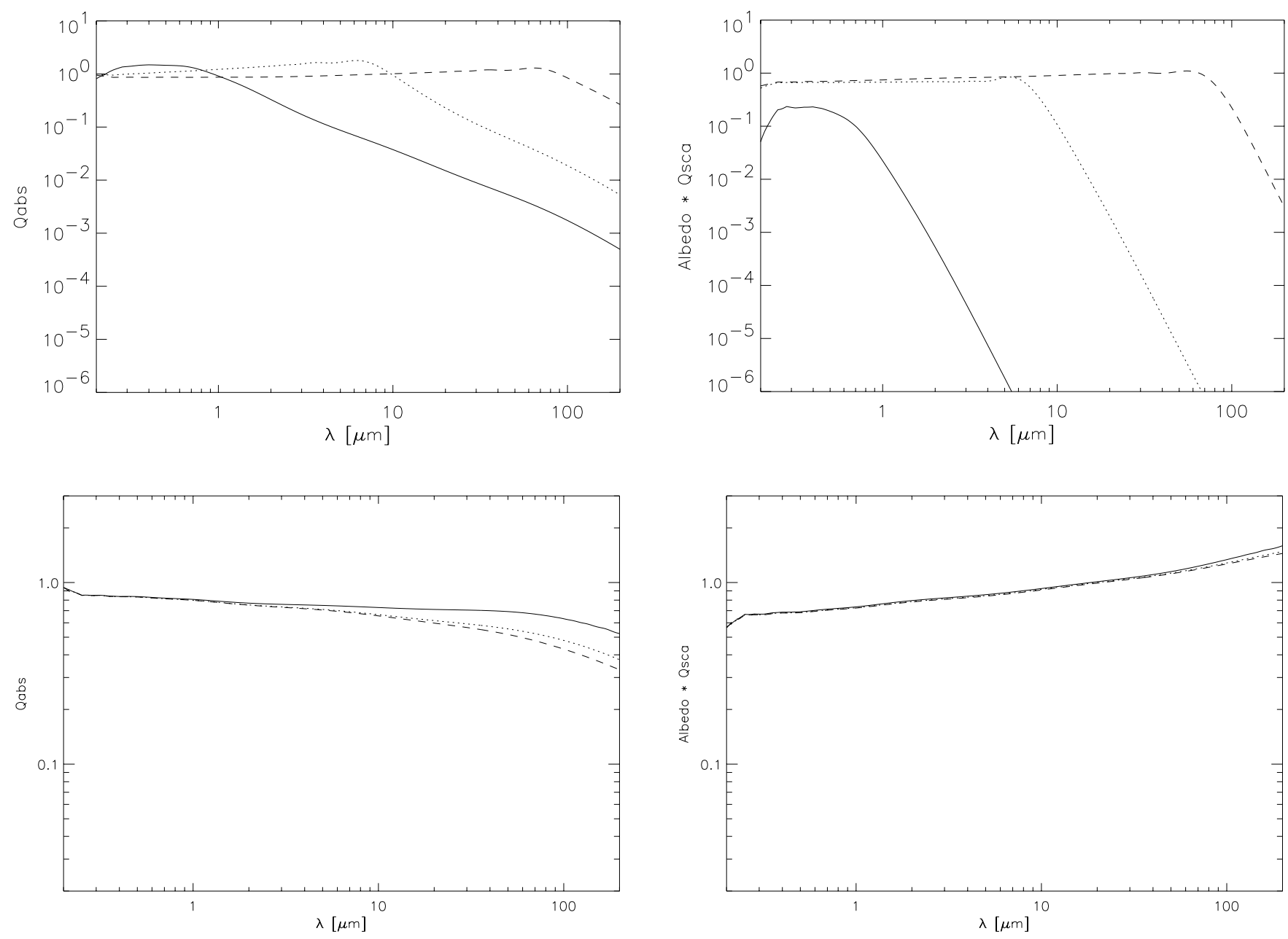

FIG. 7.-Absorption and scattering coefficients for carbon: $1000 \mathrm{~K}$ configuration ("graphite rich "). Lines are the same as in Fig. 3.

sources and the likelihood of collisional events. Since these parameters are not necessarily correlated with the actual disk size, submillimeter/millimeter measurements are required to trace larger grains that are less affected by the radiation pressure and Poynting-Robertson effect. The question to be answered here is whether there are significant differences in the SED resulting from different disk sizes.

As a result of our simulations, Figure 11 shows the SED for disks with outer radii between 25 and $400 \mathrm{AU}$. The influence on the NIR/MIR wavelength range is seen only as a decrease of the net flux with increasing disk size, which is due to the decrease of the mean temperature of the disk. At several tens of microns and beyond $100 \mu \mathrm{m}$ also the shape of the SED is affected because the smaller mean temperature results in an increase of the (sub)millimeter-to-NIR/MIR flux ratio.

\subsection{Grain Size Distribution}

In this section we consider dust models with a grain size distribution that is characterized by a (minimal) set of three parameters: the minimum and maximum grain radius $\left(a_{\min }\right.$, $\left.a_{\max }\right)$ and a power-law exponent $p$ of the size distribution:

$$
n(a) \propto a^{-p} .
$$

Since debris disks are assumed to be a place of continuous dust production as a result of collisional processes between larger particles (rather than being nurtured from a hostile interstellar medium; see, e.g., Artymowicz \& Clampin 1997), all three parameters depend on the particular debris disk system. In contrast to the interstellar medium or disks around very young stellar objects, all three parameters may therefore vary in a wide range even between different radial zones within one and the same disk (Gor'kavyi et al. 1997). Furthermore, the collision rate is assumed to decrease as the disk ages since the fraction of disk material present in larger bodies increases with time (see, e.g., Kokubo \& Ida 2002). This might allow us to classify debris disks based on their grain size distribution, at least on a statistical basis.

Constraints on the minimum grain size $a_{\mathrm{min}}$.- The smallest grain size expected to be present is determined on the one hand by the likelihood of collisional processes in the disk and on the other hand by the optical properties of the dust and the strength of the stellar radiation field. If no collisions are occurring, the minimum grain size can be estimated from the ratio of the radiation pressure to gravity, also known as $\beta$ (Burns et al. 1979),

$$
\beta=\frac{3}{16 \pi} \frac{L_{s} Q_{\mathrm{pr}}}{G M_{s} c a \rho_{g}},
$$

where $L_{s} / M_{s}$ is the central stellar luminosity/mass, $G$ is the gravitational constant, $c$ is the speed of light, $\rho_{q}$ is the 

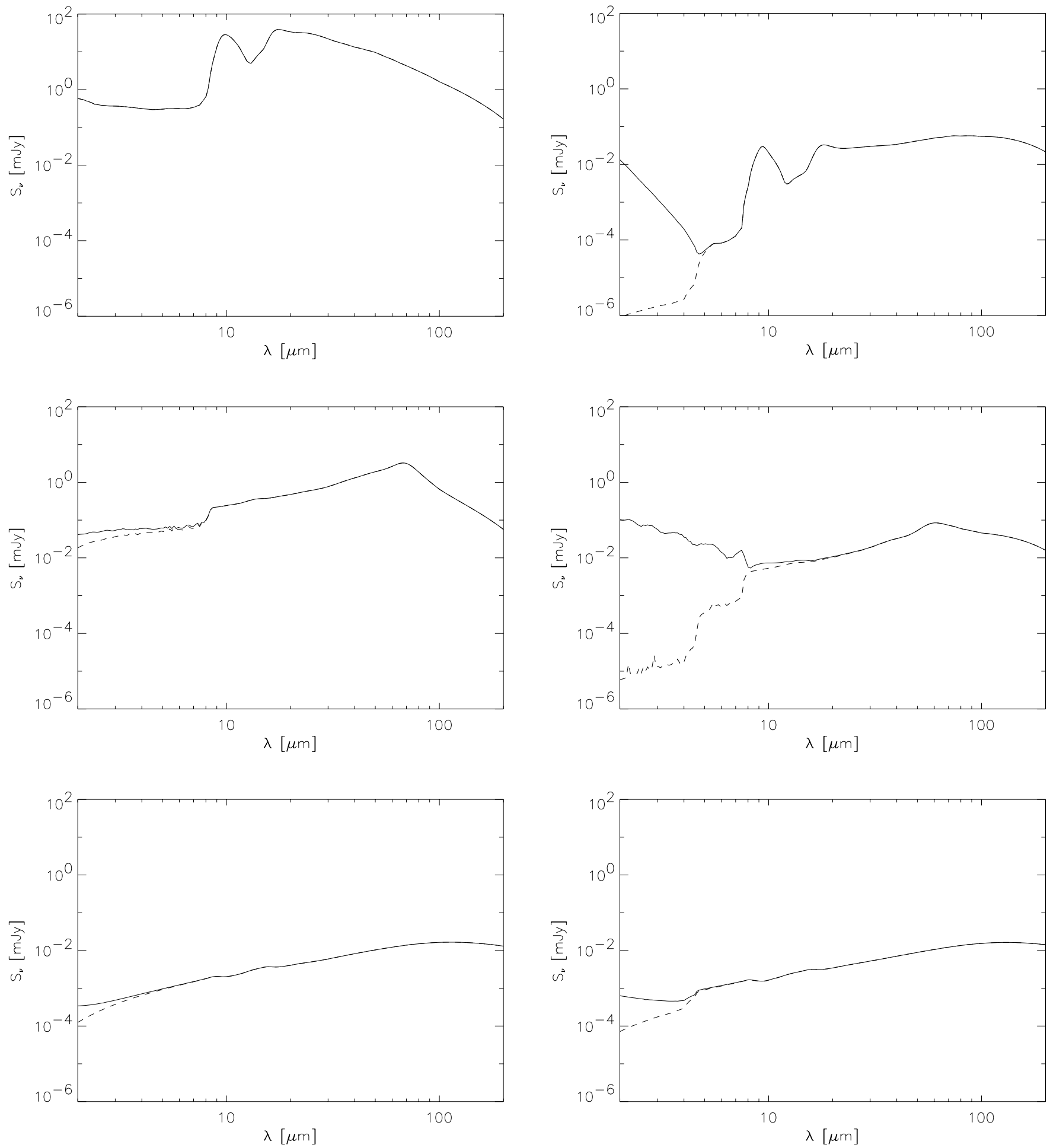

Fig. 8.- Illustration of the relative contributions of light scattering and thermal equilibrium emission in the emergent SED. Solid line: Scattered plus reemitted radiation; dashed line: reemitted radiation only. Left panels: Fe-rich silicate (amorphous olivine, $\mathrm{MgFeSiO}_{4}$ ). Right panels: Fe-poor silicate $\left(\mathrm{MgSiO}_{3}\right)$. Top: $a=0.1 \mu \mathrm{m}$. Middle: $a=10 \mu \mathrm{m}$. Bottom: $a=1 \mathrm{~mm}$. Disk mass is $10^{-10} M_{\odot}$.

material density of the grain, and $Q_{\mathrm{pr}}$ is the radiation pressure coefficient averaged over the entire stellar spectrum. Those grains for which $\beta>1$ are "blown away" by radiation pressure on a timescale much shorter than the age of the star (e.g., Aumann et al. 1984). MIR observations of the $\beta$ Pic disk by Weinberger et al. (2003) show that there must exist a substantial amount of grains with radii $\leq 10 \mu \mathrm{m}$ within 20 AU, while Pantin et al. (1997) even constrain the minimum grain size to be as small as $\approx 0.1 \mu \mathrm{m}$. In the case of Fomalhaut's debris disk, Wyatt \& Dent (2002) derive a minimum grain size of $\approx 7 \mu \mathrm{m}$ from submillimeter observations. In our parameter study we consider $a_{\min }=0.1$ and $10 \mu \mathrm{m}$ since having the bulk of the mass in larger grains does not produce a substantial MIR contribution to the SED.

Constraints on the maximum grain size $a_{\max }$ - -The upper grain size is a very uncertain parameter since 

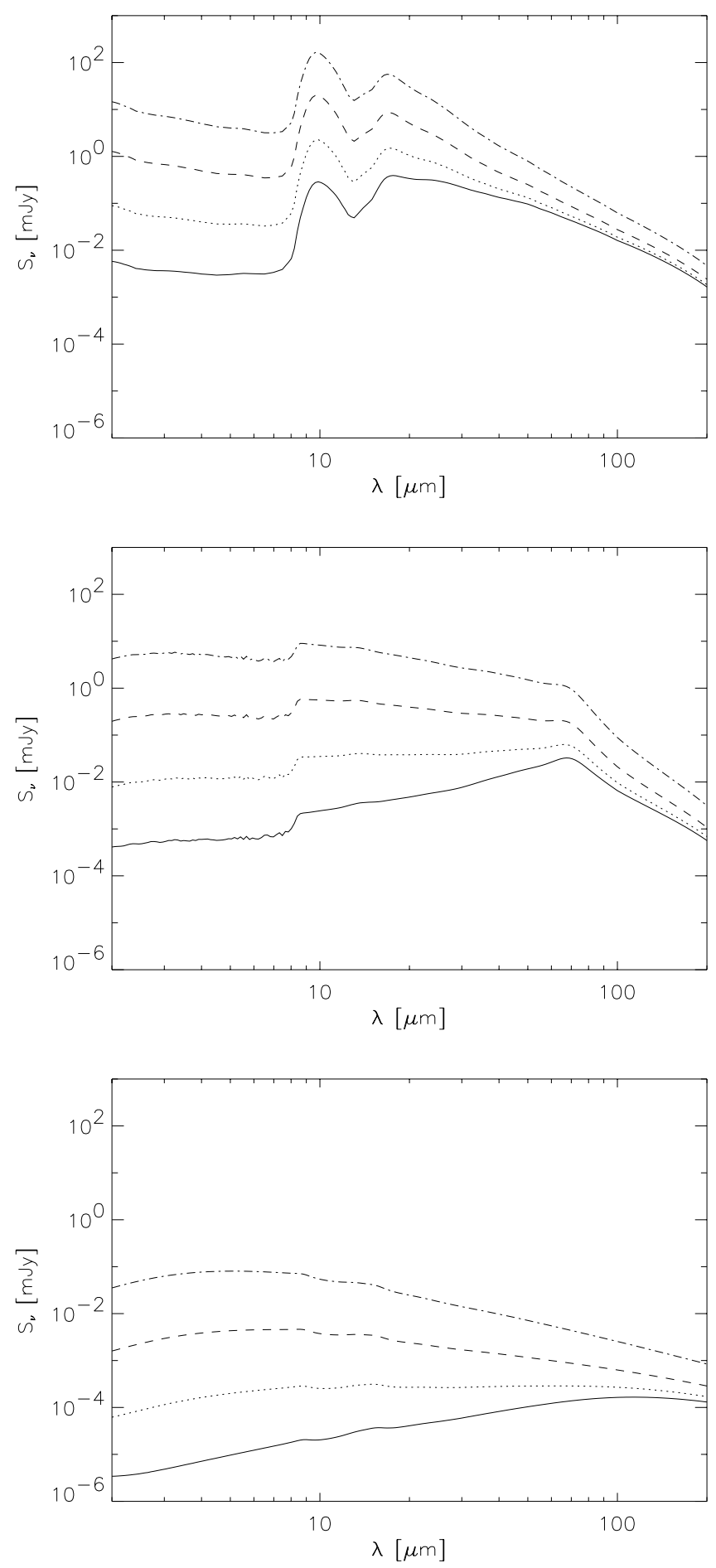

FIG. 9.-Dependence of the emergent SED on the disk density distribution. Disk density profile: $q=1.0$ (solid line), 1.5 (dotted line), 2.0 (dashed line), and 2.5 (dot-dashed line). Top: $a=0.1 \mu \mathrm{m}$. Middle: $a=10$ $\mu \mathrm{m}$. Bottom: $a=1 \mathrm{~mm}$. All calculations use amorphous olivine $\left(\mathrm{MgFeSiO}_{4}\right)$ composition and a disk mass of $10^{-12} M_{\odot}$.

debris disks are expected to harbor dust "particles" ranging from (sub)micron-sized grains to planetesimals/ planets as the result of an (almost) finished process of dust grain growth, planetesimal formation, and simultaneous dust production due to collision events (see, e.g., Beckwith, Henning, \& Nakagawa 2000). However, according to the wavelength range considered, grains larger than $\approx 1 \mathrm{~mm}$ will not give a remarkable contribution to the SED. The existence of these grains can therefore cannot be proven or ruled out by SIRTF measurements.

Complementary ground-based (sub)millimeter observations can in principal increase this upper grain size limit by about 1 order of magnitude. Based on previous observations of the very limited number of debris disks, only very weak constraints on upper grain sizes could be derived. Paresce \& Burrows (1987), for example, found an absence of significant color excess in visible observations at the outer part of the $\beta$ Pic disk, which implies that the dominant particles for the scattering are larger than a few microns. Furthermore, observations of main-sequence and pre-mainsequence (post-Herbig Ae/Be, post-T Tauri) stars show that the distribution is broad and extends from below $1 \mu \mathrm{m}$ to at least $\approx 1 \mathrm{~mm}$ (see, e.g., Skinner, Barlow, \& Justtanont 1992; Sylvester et al. 1996; Sylvester \& Skinner 1996; Li \& Greenberg 1998). In our parameter study we consider $a_{\max }=0.1,1$, and $10 \mathrm{~mm}$.

Constraints on the grain size distribution exponent $p$.-The equilibrium size distribution resulting from a collisional cascade can be described by a power law (eq. [10]) with the exponent $p=3 p_{d}-2$ (Dohnanyi 1969). Furthermore, Tanaka, Inaba, \& Nakazawa 1996 could show that $p_{d}=1.833(p=3.499)$ describes the case of a self-similar (and therefore infinite) collisional cascade. Wyatt \& Dent (2002) find in fact a very good agreement of the dust SED from Fomalhaut's debris disk with the assumption of $p=3.499$ (see also Holland et al. 2003). However, in general, real dust particle/planetesimal ensembles are expected to be characterized by size-dependent particle strengths, therefore resulting in a distribution of exponents $p$ as a function of grain size. The "mean" size distribution exponent could then be slightly shifted from 3.499 (Durda \& Dermott 1997). For example, Pantin et al. (1997) derived two different exponents to fit MIR images of the $\beta$ Pic disk: $p=3.0$ and 3.3 for the smallest grains (with radii up to $10 \mu \mathrm{m}$ ) and the larger population, respectively. Since particle growth/ collision processes are expected to be mainly dependent on the radial distance from the star (Weidenschilling \& Davis 2000), the dependence on the (mean) grain size translates into a dependence of the exponent $p$ on the location within the disk. Another argument for a clear deviation from $p=3.499$ comes from the fact that newly created small grains are likely to be removed from the system faster than larger ones may decrease this value, at least on short timescales, i.e., as long as a small grain population resulting from collision processes is present (see, e.g., Moro-Martín $\&$ Malhotra 2003). We consider two different values of the exponent, $p=2.5$ and 3.5 .

In Figure 12 the SEDs resulting from dust disks with different grain size distributions are shown. Based on the discussion above, we consider the following parameter combinations: $a_{\min }=0.1$ and $10 \mu \mathrm{m} ; a_{\max }=0.1,1$, and $10 \mathrm{~mm}$; and $p=2.5$ and 3.5. Comparing the SEDs within these parameter ranges and the considered wavelength range, one can draw the main conclusion that the relative number of small grains in the system (determined by $a_{\min }, a_{\max }$, and $p$ ) influences the shape of the SED. In particular, we find the following:

1. Increasing the maximum grain size results in a decrease of the net flux and a decrease of dust compositionspecific emission features. Since the amount of the underlying continuum can be due to either almost featureless 

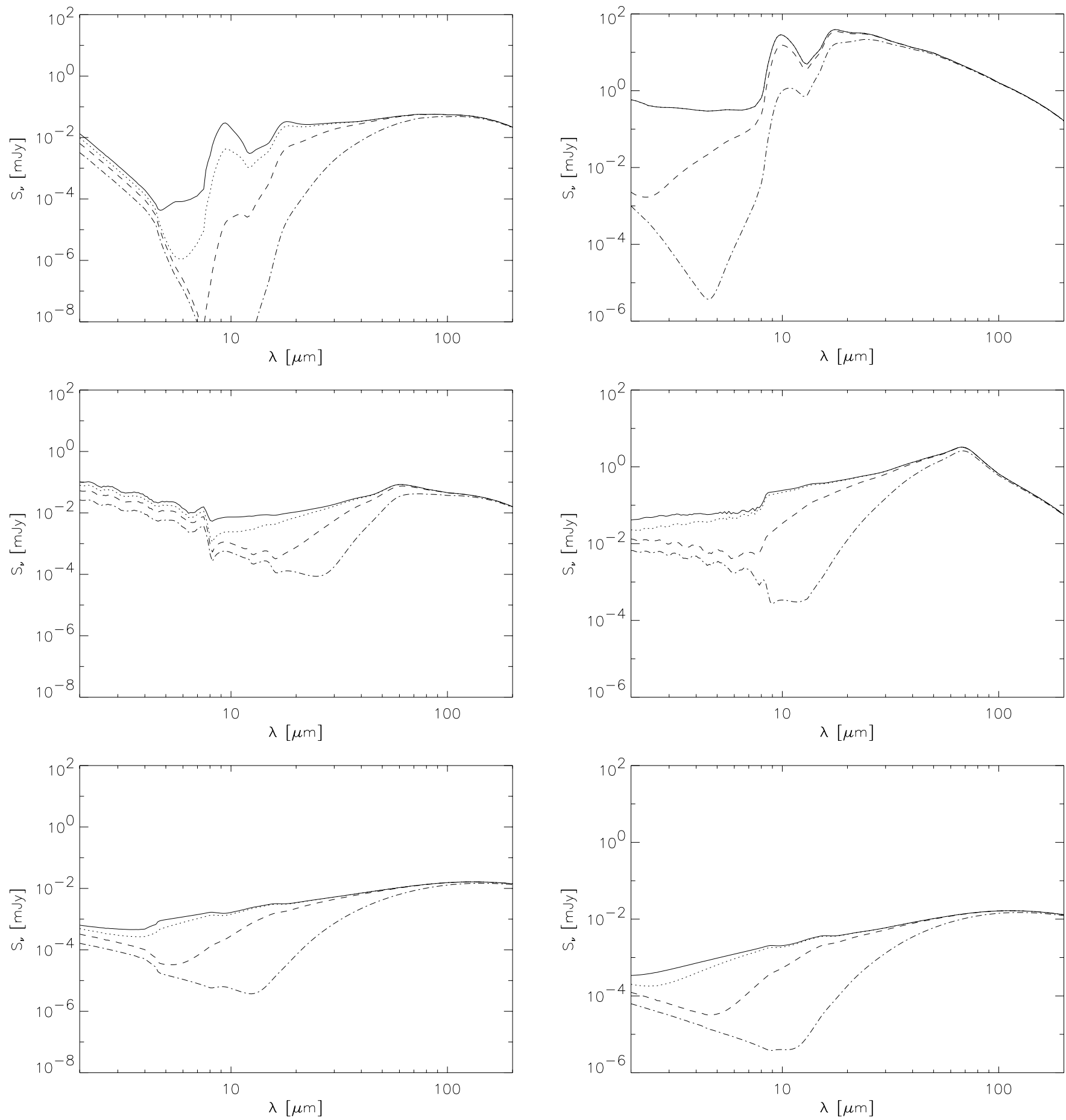

FIG. 10.--Influence of inner gaps on the SED. Inner disk radius: dust sublimation radius (solid line), $0.1 \mathrm{AU}$ (dotted line), $1 \mathrm{AU}$ (dashed line), and $10 \mathrm{AU}$ (dot-dashed line). Left panels: Fe-poor silicate $\left(\mathrm{MgSiO}_{3}\right)$. Right panels: Fe-rich (amorphous olivine, $\left.\mathrm{MgFeSiO}_{4}\right)$. Top: $a=0.1 \mu \mathrm{m}$. Middle: $a=10 \mu \mathrm{m}$. Bottom: $a=1 \mathrm{~mm}$. Assuming a dust sublimation temperature of $1550 \mathrm{~K}$, the sublimation radius of $\mathrm{MgSiO}_{3} / \mathrm{MgFeSiO}_{4}$ amounts to $7.3 \times 10^{-3} \mathrm{AU} / 1.3 \times 10^{-1} \mathrm{AU}$ $(a=0.1 \mu \mathrm{m}), 1.2 \times 10^{-2} \mathrm{AU} / 3.5 \times 10^{-2} \mathrm{AU}(a=10 \mu \mathrm{m})$, and $3.3 \times 10^{-2} \mathrm{AU} / 3.2 \times 10^{-2} \mathrm{AU}(a=1 \mathrm{~mm})$. Disk mass is $10^{-10} M_{\odot}$.

emission by carbon grains of arbitrary size (distribution) or large silicate grains, the maximum grain size might become the most difficult parameter to be derived from SIRTF measurements alone. However, millimeter measurements can be used to distinguish between the scenarios based on different slopes of the SED (similar to what has been done in the case of T Tauri disks; see Beckwith \& Sargent 1991).

2. Given a fixed disk mass, increasing the grain size distribution exponent and decreasing the minimum grain size increases the relative amount of small grains and therefore pronounced dust-specific spectral features in the SED.

The SEDs shown in Figure 12 illustrate that the relative abundance of small grains and therefore the minimum grain size and grain size distribution exponent will be wellconstrained disk parameters from SIRTF measurements as a result of the abundance of spectral features in the 5-40 $\mu \mathrm{m}$ range (see also Figs. 3, 4, and 5). 

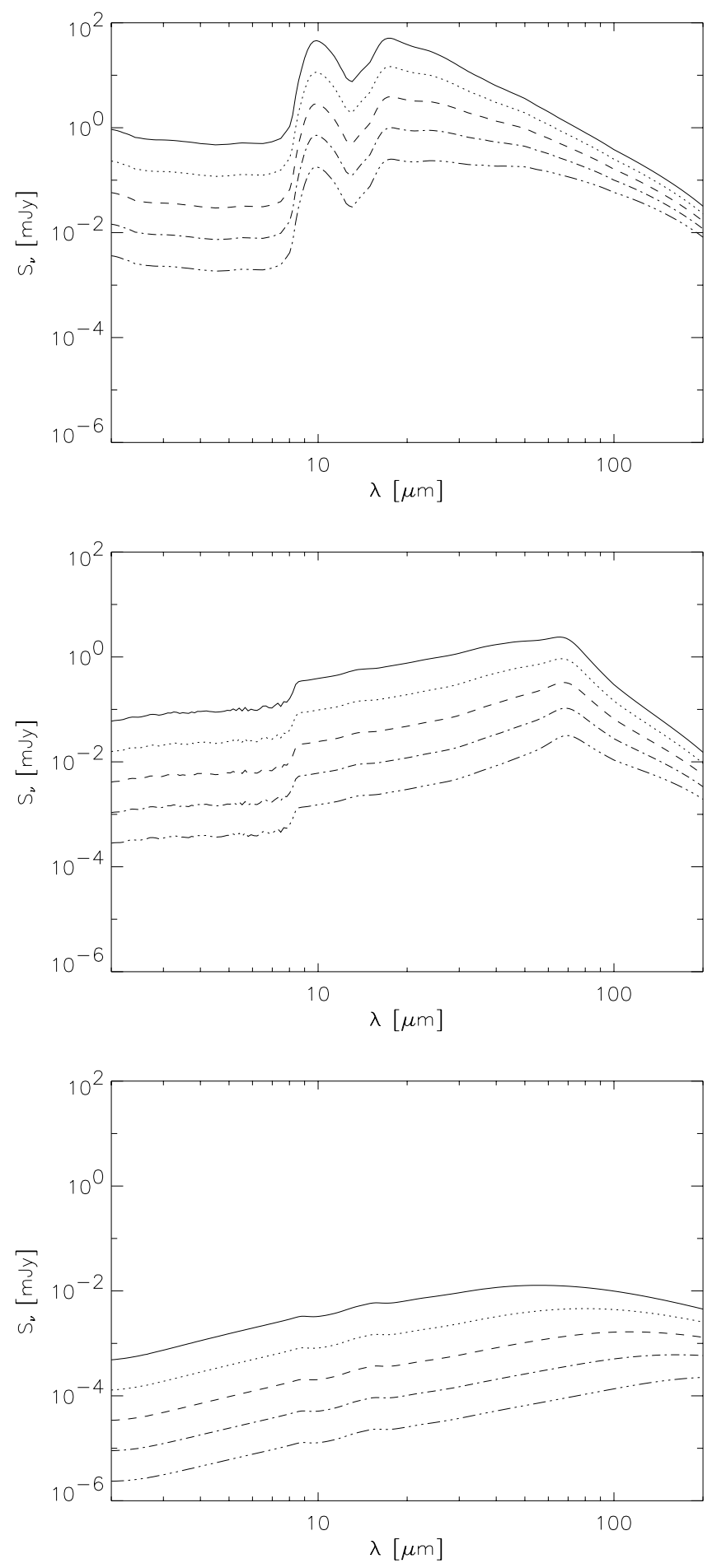

FIG. 11.- Influence of the outer disk radius on the grain size distribution. Outer radius of the disk: 25 (solid line), 50 (dotted line), 100 (dashed line), 200 (dot-dashed line), and $400 \mathrm{AU}$ (triple-dot-dashed line). Grain radius: $0.1 \mu \mathrm{m}$ (top), $10 \mu \mathrm{m}$ (middle), $1 \mathrm{~mm}$ (bottom). Amorphous olivine $\left(\mathrm{MgFeSiO}_{4}\right)$ composition and a disk mass of $10^{-11} M_{\odot}$ are assumed.

\section{SED PROBABILITY DISTRIBUTION}

In this section we assess the likelihood of observing a particular SED given an assumed underlying distribution of the parameters we have varied in the previous subsections. We consider a plausible range of values for the minimum and maximum grain size, the radial density distribution expo- nent $q$, and the grain size distribution exponent $p$. The other disk and dust parameters are fixed: the inner radius (equal to the sublimation radius), the outer radius ( $=100 \mathrm{AU})$, and the chemical composition of the dust (amorphous olivine, $\mathrm{MgFeSiO}_{4}$ ). According to the previous parameter range justifications for the variable quantities, we calculate first the separate SEDs for $10^{3}$ grain sizes logarithmically distributed between 0.1 and $1000 \mu \mathrm{m}$ in disks with 31 different radial density distribution exponents linearly distributed in the range [1.0, 2.5], i.e., $3.1 \times 10^{4}$ separate SEDs. In the second step we select a lower and upper grain size randomly from this grain size distribution, assuming an equipartition of grain sizes on the prepared logarithmic grid of possible grain radii. Furthermore, we randomly chose the grain size distribution exponent $p$ from one of 11 possible values linearly distributed in the range $[2.5,3.5]$ and one of the 31 radial density distribution exponents $q$. In the third step we derive the net SED for a disk defined by these randomly chosen parameter settings based on the linear weighted combination of the single SEDs calculated in the first step. In order to allow the comparison of the (shapes of) SEDs resulting from the $31 \times 11 \times \sum_{i=1}^{1000} i \approx 1.7 \times 10^{8}$ possible different disks, the net luminosity is normalized to " 1 " (arbitrary unit). For this reason, the single SEDs had to be calculated over a larger parameter range than in the previous studies in order to minimize neglected flux reemission appearing outside the considered wavelength range; here we chose $\lambda=[0.2 \mu \mathrm{m}, 4000 \mu \mathrm{m}]$. In Figure 13 the distribution of $1.2 \times 10^{7}$ normalized, individual disk SEDs is shown, whereby the density of SEDs in the flux-wavelength space is color coded.

This SED density distribution of course depends on the particular distribution of dust and disk parameters within the given parameter ranges. In order to illustrate this, in Figure 13 a second set of SED density distributions is shown for which the minimum and maximum grain sizes have been randomly selected from a linear distribution of grain sizes (instead of from a logarithmic one as before). The main difference between the approaches is the much stronger pronunciation of the silicate emission bands in the case of a logarithmic distribution since in that case both the mean minimum and mean maximum selected grain sizes are smaller than in the case of a linear grid of possible grain size radii. The "real" (unknown) distribution of possible grain size boundaries depends on the outcome of collision processes. The comparison of observed SED density distributions with distributions as shown in Figure 13 but based on theoretically predicted collision scenarios may therefore provide a key to prove the underlying physics of collision processes in debris disks.

The range in normalized flux density, however, over which possible debris disk SEDs are distributed does depend on the parameter range of the dust and disk parameters only and is therefore the same in both approaches. Our simulations show that in the case of a possible grain size distribution in the range of $0.1-1000 \mu \mathrm{m}$ the most narrow distribution of already normalized SEDs still spans a range over more than 1 order of magnitude in possible fluxes (in the range of $\lambda \approx 40-50 \mu \mathrm{m}$ ) but increases to above 3 orders of magnitude toward the lower and upper boundaries of the considered wavelength interval. The scattering of the SEDs slightly decreases if the range of possible grain radii is decreased (see also Fig. 13). In particular, we find an 

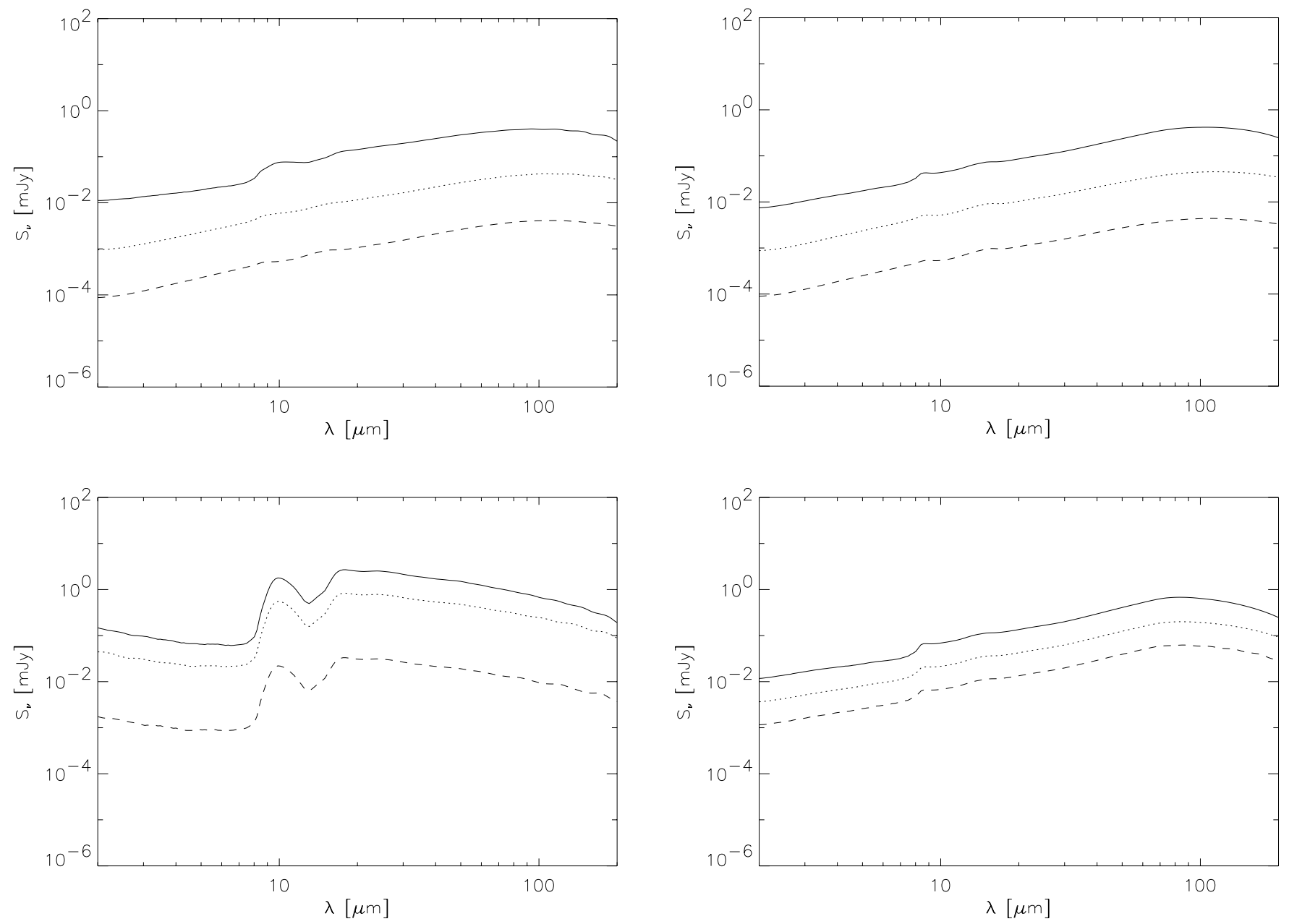

Fig. 12.-Influence of the grain size distribution on the SED. Grain size distribution exponent: $p=2.5$ (top) and 3.5 (bottom). Minimum grain size: $a_{\min }=0.1($ left $)$ and $10 \mu \mathrm{m}($ right $)$. Maximum grain size: $a_{\max }=0.1$ (solid line), 1 (dotted line), and $10 \mathrm{~mm}$ (dashed line). Amorphous olivine (MgFeSiO 4 ) composition and a disk mass of $10^{-10} M_{\odot}$ are assumed.

additional minimum of possible flux values around $\lambda \approx 50-70 \mu \mathrm{m}$ for our particular dust/disk setup in the case of large grain size distributions $(a=10-1000 \mu \mathrm{m})$. This spectral regime may be the most diagnostic for determining disk masses relatively independent of the details of the assumed disk or grain properties.

\section{SIRTF COLORS}

Based on selected SEDs presented in previous sections, we derive the colors $m(8 \mu \mathrm{m})-m(24 \mu \mathrm{m})$ and $m(24 \mu \mathrm{m})-m(70$ $\mu \mathrm{m})$, applying the filter characteristics of the SIRTF cameras IRAC $(8 \mu \mathrm{m})$ and MIPS (24 and $70 \mu \mathrm{m})$. The resulting colorcolor diagrams, shown in Figures 14 and 15 (see also Fig. 16), are normalized to a blackbody with a temperature of $9650 \mathrm{~K}$, which sufficiently accurately represents the MIR-tosubmillimeter SED of the A0 V standard star Vega (Castelli \& Kurucz 1994; Decin et al. 2003):

$$
\begin{aligned}
m_{1}-m_{2}= & -2.5\left(\log _{10} \frac{\int_{0.2 \mu \mathrm{m}}^{200 \mu \mathrm{m}} T_{1} S_{\lambda} d \lambda}{\int_{0.2 \mu \mathrm{m}}^{200 \mu \mathrm{m}} T_{2} S_{\lambda} d \lambda}\right) \\
& +\left(m_{1, \text { standard }}-m_{2, \text { standard }}\right)
\end{aligned}
$$

where

$$
\begin{aligned}
m_{1, \text { standard }}- & m_{2, \text { standard }} \\
& =-2.5 \log _{10} \frac{\int_{0.2 \mu \mathrm{m}}^{200 \mu \mathrm{m}} T_{1} B_{\lambda}(9650 \mathrm{~K}) d \lambda}{\int_{0.2 \mu \mathrm{m}}^{200 \mu \mathrm{m}} T_{2} B_{\lambda}(9650 \mathrm{~K}) d \lambda} .
\end{aligned}
$$

Here $S_{\lambda}=S_{\nu} d \nu / d \lambda$ is the dust scattering/reemission SED, $T_{1}$ and $T_{2}$ describe the wavelength-dependent filter transmission, ${ }^{6}$ and $B_{\lambda}$ is the Planck function. Note in these figures that only the dust emission/scattering spectrum is represented; the stellar photosphere has been removed in the SEDs.

These diagrams reflect what has been outlined in the discussions about the possibility to distinguish between different disk models. For example, the color-color diagram for disks with different outer radii (Fig. 15, top right-hand panel, based on the SEDs given in Fig. 11) shows a large dynamic range of colors and therefore clearly distinguishable models only in the case of small dust grains (here 0.1

\footnotetext{
${ }^{6}$ SIRTF instrument characteristics are available at http://sirtf.caltech.edu/SSC/obs.
} 

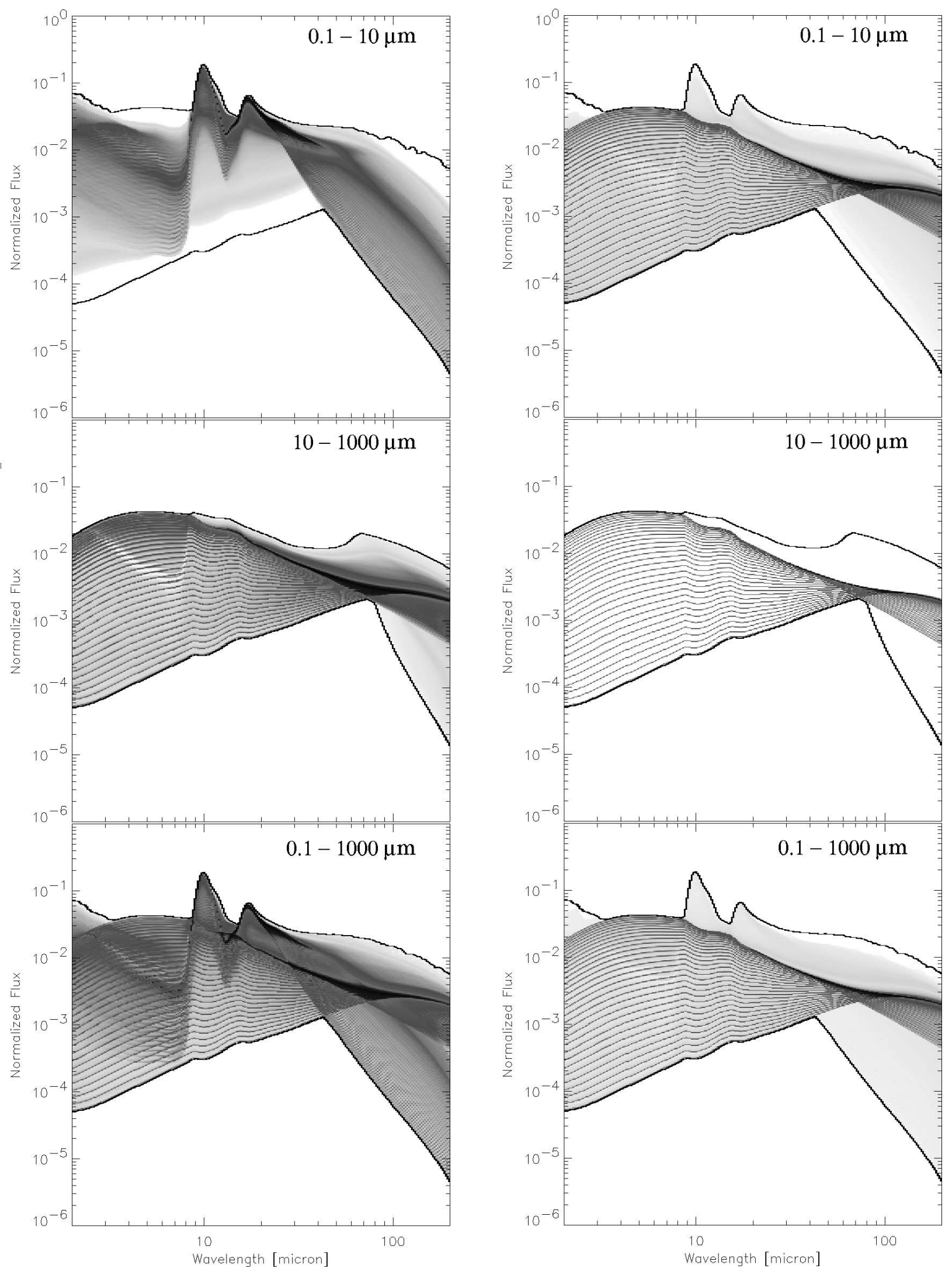

FIG. 13.-Distribution of normalized debris disk SEDs in the range [0.2 $\mu \mathrm{m}, 200 \mu \mathrm{m}]$. Ranges of variable disk parameters: $p=[2.5,3.5], q=[1.0,2.5]$. The particle size ranges from which the minimum and maximum grain sizes are randomly selected are given in each figure. Minimum and maximum grain sizes are selected from a logarithmically equidistant/linear distribution of grain sizes in the left/right-hand panels. Color coding: dark regions represent a high SED density, while light-gray regions symbolize low SED densities. The black upper and lower curves in each figure mark the range within which solutions (SEDs) were found. 


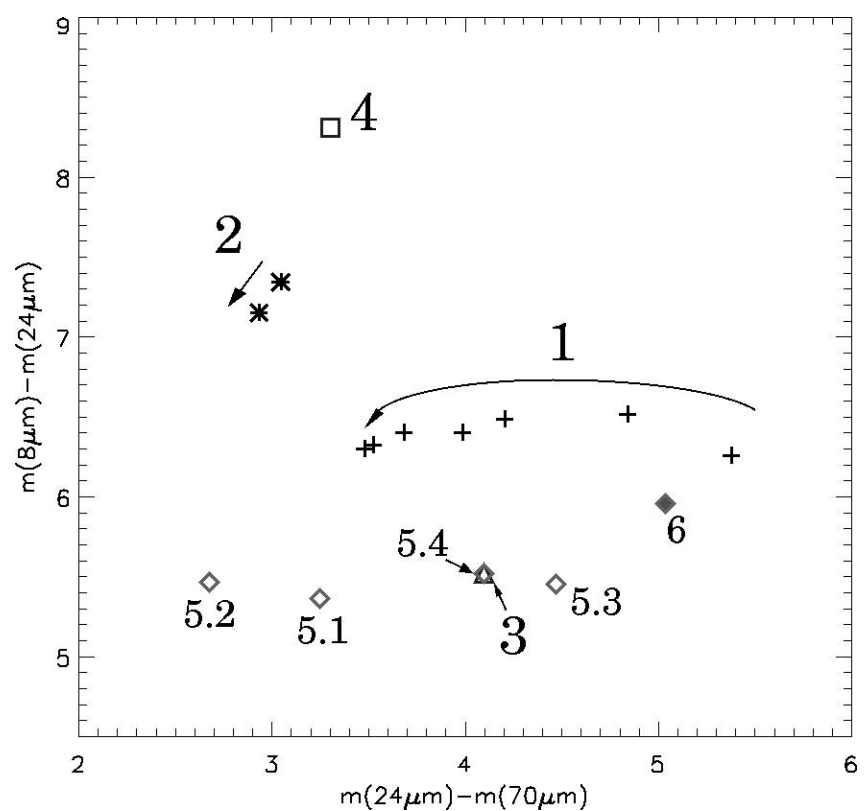

FIG. 14.-SIRTF color-color diagrams corresponding to the SEDs shown in Fig. 2. (1) $\mathrm{MgSiO}_{3} \rightarrow \mathrm{Mg}_{0.95} \mathrm{Fe}_{0.05} \mathrm{SiO}_{3} \rightarrow \mathrm{Mg}_{0.8} \mathrm{Fe}_{0.2} \mathrm{SiO}_{3} \rightarrow$ $\mathrm{Mg}_{0.7} \mathrm{Fe}_{0.3} \mathrm{SiO}_{3} \rightarrow \mathrm{Mg}_{0.6} \mathrm{Fe}_{0.4} \mathrm{SiO}_{3} \rightarrow \mathrm{Mg}_{0.5} \mathrm{Fe}_{0.5} \mathrm{SiO}_{3} \rightarrow \mathrm{Mg}_{0.4} \mathrm{Fe}_{0.6} \mathrm{SiO}_{3}$. (2) $\mathrm{MgFeSiO}_{4} \rightarrow \mathrm{Mg}_{0.8} \mathrm{Fe}_{1.2} \mathrm{SiO}_{4}$. (3) "Astronomical silicate." (4) Crystalline olivine. (5) Carbon: (5.1) C (400 K), (5.2) C (600 K), (5.3) C (800 K), (5.4) C (1000 K). (6) Graphite.

$\mu \mathrm{m})$. In contrast to this, in a grain size distribution with grain radii of $10 \mu \mathrm{m}$ and $1 \mathrm{~mm}$ the almost constant offset between the SEDs in the MIR wavelength range results in a nearly constant value of $m(8 \mu \mathrm{m})-m(24 \mu \mathrm{m})$.

\section{SUMMARY AND CONCLUSIONS}

In preparation for SIRTF and SOFIA observations of circumstellar debris disks we have investigated the influence of various disk and dust parameters on the resulting SED, in particular the offset from the stellar photospheric flux due to dust reemission and scattering processes. Based on an analytic disk model, we considered parameter ranges as suggested from existing observations of a small number of debris disk systems, as well as constraints derived from theoretical approaches. We restricted our investigations primarily to the wavelength range accessible by SIRTF .

We draw the following main conclusions from our study of the parameter space:

1. The correct estimation/subtraction of the stellar photospheric flux is essential for the subsequent data analysis in two respects. First, it is important for the correct estimation of the dust continuum (not taking into account any particular dust emission features) that mainly constrains the disk mass, the upper grain size and grain size distribution exponent ( $(4.4)$, the outer disk radius $(\S 4.3)$, and the overall radial disk density distribution exponent (§ 4.1). Secondly, the strength of remaining stellar spectral features allows one to derive conclusions about the scattering to absorption efficiency (or albedo) of the dust grains and therefore, based on a simple silicate/carbon dust grain model, to derive constraints on the crystalline silicate/iron-poor amorphous silicate to iron-rich amorphous silicate/carbon ratio $(\S 3)$.

2. The characteristic amorphous silicate broadband solid-state emission features are predicted to be of decisive importance for the characterization of the evolutionary state of debris disks. The appearance of these features depends strongly on the existence of small grains (smaller than about $10 \mu \mathrm{m}$ ), the abundance of which, however, is assumed to be small in a collisionless system (depending on the stellar SED; $\S \S 3$ and 4.4). The strength of these features will allow us to decide if collision processes took place still recently in the disk of the particular star. However, one has to take into account that only "snapshots" of the current (with respect to the timescale of the collisional events), arbitrarily selected states of debris disks can be obtained. The SED and thus the derived smallest size of highly abundant grains do therefore not necessarily describe the real, "mean" evolutionary state of a particular disk (if defined by the time-dependent collision rate). However, surveys based on a statistically large number of debris disk systems with large subsets of stars with a similar age will allow us to derive general characteristics of debris disks as a function of their (evolutionary) age.

3. The MIR continuum will allow us to conclude if an inner hole, due to the presence of at least one embedded planet, exists. Furthermore, the inner radius of that gap (equal to the orbit of the planet) could be derived (§ 4.2).

The presented study was performed to address the most fundamental questions one will want to answer with the analysis of the SED of debris disk systems. Based on this "first-level" analysis, a more detailed investigation of particular debris disk systems will be possible. For instance, the radial segregation of chemically different dust grains, the spatial distribution of ice-mantled grains with spectral features in the range of 10-100 $\mu \mathrm{m}$ (e.g., Warren 1984), the possibility to distinguish qualitatively different planetary architectures embedded in debris disks (Moro-Martín \& Malhotra 2002), and the determination of characteristic wavelength ranges in which planetary radiation (in particular astrobiological tracers) can be separated from the underlying dust continuum radiation (Beichmann 1996; Fischer \& Pfau 1997) are tasks that are beyond the recent study and will be considered in a subsequent investigation.

S. W. was supported through the NASA grant NAG 511645 and through the SIRTF legacy science program through an award issued by JPL/CIT under NASA contract 1407 . We are grateful for valuable discussions with Th. Henning, S. Weidenschilling, A. Moro-Martín, and the other members of the FEPS team led by M. Meyer. 

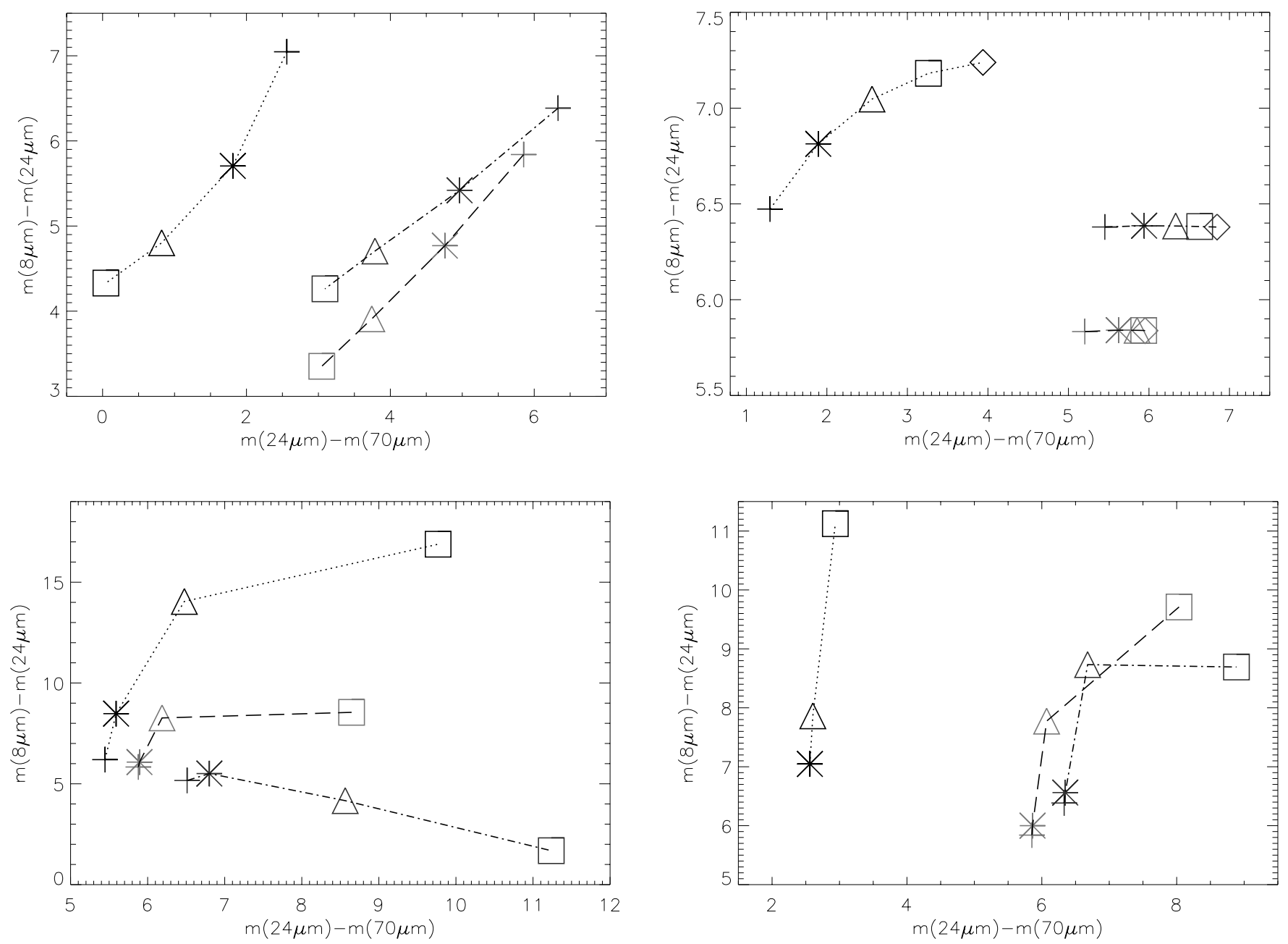

FIG. 15.-SIRTF color-color diagrams corresponding to the SEDs shown in Figs. 9 (top left), 11 (top right), and 10 (bottom). Different line styles represent different dust grain radii: $0.1 \mu \mathrm{m}$ (dotted line), $10 \mu \mathrm{m}$ (dot-dashed line), $1 \mathrm{~mm}$ (dashed line). Top left: Different symbols represent different power-law exponents $q$ of the disk density distribution: $q=1.0$ (plus sign), 1.5 (asterisk), 2.0 (triangle), and 2.5 (square). Top right: Different symbols represent different outer disk radii: 25 (plus sign), 50 (asterisk), 100 (triangle), 200 (square), and $400 \mathrm{AU}$ (rhombus). Bottom left/right: Different symbols represent different inner gap radii for the case of $\mathrm{MgSiO}_{3} / \mathrm{MgFeSiO}_{4}$ grains: sublimation radius (plus sign), $0.1 \mathrm{AU}$ (asterisk), 1.0 AU (triangle), and $10 \mathrm{AU}$ (square).
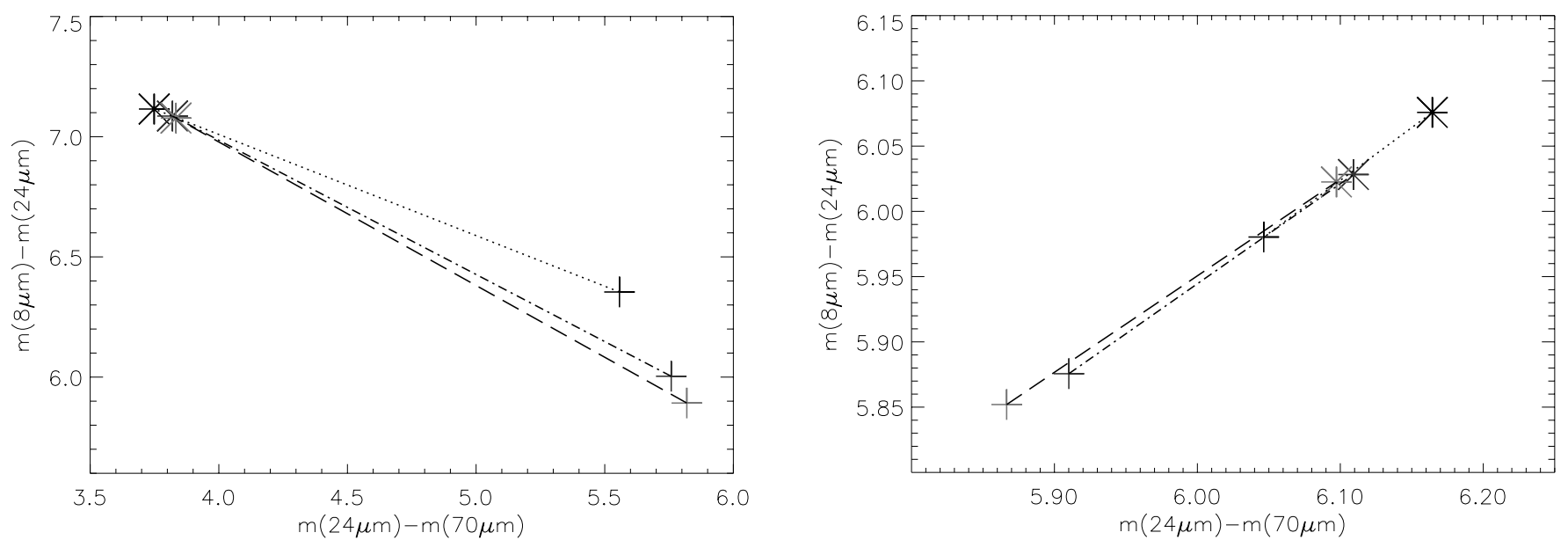

FIG. 16. - SIRTF color-color diagrams corresponding to the SEDs shown in Fig. 12. Left: Minimum grain radius amounts to $0.1 \mu \mathrm{m}$. Right: Minimum grain radius amounts to $10 \mu \mathrm{m}$. Different line styles represent different maximum dust grain radii: 0.1 (dotted line), 1 (dot-dashed line), and $10 \mathrm{~mm}$ (dashed line). Different symbols represent different power-law exponents $p$ of the grain size distribution: $p=2.5$ ( plus sign) and $q=3.5$ (asterisk). 
Artymowicz, P. 1997, Annu. Rev. Earth Planet. Sci., 25, 175

Artymowicz, P., Burrows, C., \& Paresce, F. 1989, ApJ, 337, 494

Artymowicz, P., \& Clampin, M. 1997, ApJ, 490, 863

Aumann, H., et al. 1984, ApJ, 278, L23

Beckwith, S. V. W., Henning, Th., \& Nakagawa, Y. 2000, in Protostars \& Planets IV, ed. V. Manning, A. P. Boss, \& S. S. Russel (Tucson: Univ. Arizona Press), 533

Beckwith, S. V. W., \& Sargent, A. I. 1991, ApJ, 381, 250

Beichmann, C. 1996, JPL Publ. 96-22, A Road Map for the Exploration of Neighboring Planetary Systems (ExNPS) (Pasadena: JPL)

Bouwman, J., De Koter, A., van den Anker, M. E., \& Waters, L. B. F. M. 2000, A\&A, 360, 213

Briggs, R. E. 1962, AJ, 67, 710

Bryden, G., Chen, X., Lin, D. N. C., Nelson, R. P., \& Papaloizou, J. C. B. 1999, ApJ, 514, 344

Bryden, G., Rózyczka, M., Lin, D. N. C., \& Bodenheimer, P. 2000, ApJ, 540,1091

Burns, J. A., Lamy, P. L., \& Soter, S. 1979, Icarus, 40, 1

Burrows, C. J., et al. 1996, ApJ, 473, 437

Butler, R. P., Marcy, G. W., Williams, E., Hauser, H., \& Shirts, P. 1997, ApJ, 474, L115

Calvet, N., D'Alessio, P., Hartmann, L., Wilner, D., Walsh, A., \& Sitko, M. 2002, ApJ, 568, 1008

Campbell, M. F., et al. 1976, ApJ, 208, 396

Castelli, F., \& Kurucz, R. L. 1994, A\&A, 281, 817

Chiang, E. I., \& Goldreich, P. 1999, ApJ, 519, 279

Chrysostomou, A., et al. 2000, MNRAS, 312, 103

Cotera, A. S., et al. 2001, ApJ, 556, 958

D’Alessio, P., Calvet, N., Hartmann, L., Lizano, S., \& Cantó, J. 1999, ApJ, 527,893

Decin, L., et al. 2003, A\&A, 400, 695

Dent, W. R. F., Greaves, J. S., Mannings, V., Coulson, I. M., \& Walther, D. M. 1995, MNRAS, 277, L25

Dent, W. R. F., Walker, H. J., Holland, W. S., \& Greaves, J. S. 2000, MNRAS, 314, 702

Dermott, S. F. Durda, D. D., Gustafson, B. A., Jayaraman, S., Xu, Y. L., Gomes, R. S., \& Nicholson, P. D. 1992, in Asteroids, Comets, Meteors, ed. A. W. Harris \& E. Bowell (Lunar and Planetary Inst.), 153

Dohnanyi, J. S. 1969, J. Geophys. Res., 74, 2531

Dollfus, A., \& Suchail, J.-L. 1987, A\&A, 187, 669

Dorschner, J., Begemann, B., Henning, Th., Jäger, C., \& Mutschke, H. 1995, A\&A, 300, 503

Draine, B. T., \& Lee, H. M. 1984, ApJ, 285, 89

Durda, D. D. \& Dermott, S. F. 1997, Icarus, 130, 140

Elvius, A., \& Hall, J. S. 1967, Lowell Obs. Bull., 7, 17

Epifani, E., et al. 2001, Icarus, 149, 339

Fischer, O., \& Pfau, W. 1997, A\&A, 325, 55

Gor'kavyi, N. N., Ozernoy, L. M., Mather, J. C., \& Taidakova, T. 1997, ApJ, 488, 268

Grady, C. A., et al. 2000, in Protostars and Planets IV, ed. V. Mannings,

A. P. Boss, \& S. S. Russell (Tuscon: Univ. Arizona Press), 613

Greaves, J. S., Coulson, I. M., \& Holland, W. S. 2000a, MNRAS, 312, L1

Greaves, J. S., Mannings, V., \& Holland, W. S. 2000b, Icarus, 143, 155

Greaves, J. S., et al. 1998, ApJ, 506, L133

Gürtler, J., Kömpe, C.. \& Henning, Th. 1996, A\&A, 305, 878

Habing, H. J., et al. 2001, A\&A, 365, 545

Hajjar, R., \& Bastien, P. 1996, in ASP Conf. Ser. 97, Polarimetry of the Interstellar Medium, ed. W. G. Roberge \& D. C. B. Whittet (San Francisco: ASP), 355

Henning, Th., Begemann, B., Mutschke, H., \& Dorschner, J. 1995, A\&AS, 112,143

Henning, Th., Il'In, V. B., Krivova, N. A., Michel, B., \& Voshchinnikov, N. V. 1999, A\&AS, 136, 405

Henning, Th., \& Stognienko, R. 1996, A\&A, 311, 291

Holland, W. S., et al. 1998, Nature, 392, 788

2003, ApJ, 582,1141

Ishimoto, H. 2000, A\&A, 362, 1158

Ishimoto, H., \& Mann, I. 1999, Planet. Space Sci., 47, 225

Jäger, C., Mutschke, H., Begemann, B., Dorschner, J., \& Henning, Th. 1994 A\&A, 292, 641

Jäger, C., Mutschke, H., \& Henning, Th. 1998, A\&A, 332, 291

Jayawardhana, R., Holland, W. S., Kalas, P., Greaves, J. S., Dent, W. R. F., Wyatt, M. C., \& Marcy, G. W. 2002, ApJ, 570, L93

Johnson, J. J. \& Jones, T. J. 1991, AJ, 101, 1735

Kalas, P., \& Jewitt, D. 1995, AJ, 110, 794
Kastner, J. H. \& Weintraub, D. A. 1996, in ASP Conf Ser 97, Polarimetry of the Interstellar Medium, ed. W. G. Roberge \& D. C. B. Whittet (San Francisco: ASP), 212

Kenyon, S. J., \& Bromley, B. C. 2001, AJ, 121, 538

Kenyon, S. J., \& Hartmann, L. 1987, ApJ, 323, 714

Kittel, L. 1963, Introduction to Solid State Physics (New York: Wiley)

Kley, W. 2000, MNRAS, 313, L47

Koerner, D. W., Sarget, A. I., \& Beckwith, S. V. W. 1993, Icarus, 106, 2

Kokubo, E. \& Ida, S. 2002, ApJ, 581, 666

Labs, D., \& Neckel, H. 1968, Z. Astrophys., 69, 1

Lecalvier des Etangs, A., Vidal-Madjar, A., \& Ferlet, R. 1998, A\&A, 339, 477

Li, A., \& Greenberg, J. M. 1997, A\&A, 323, 566 1998, A\&A, 331, 291

Liou, J.-C., Dermott, S. F., \& Xu, Y.-L. 1995, Planet. Space Sci., 43, 717

Liou, J.-C., \& Zook, H. A. 1999, AJ, 118, 580

Liseau, R. \& Artymowicz, P. 1998, A\&A, 334, 935

Lumme, K., \& Rahola, J. 1994, ApJ, 425, 653

Malfait, K., Waelkens, C., Bouwman, J., De Koter, A., \& Waters, L. B. F. M. 1999, A\&A, 345, 181

Marcy, G. W., Butler, R. P., Vogt, S. S., Fischer, D., \& Liu, M. 1999, ApJ, 520,239

Martin, P. G., \& Rogers, C. 1987, ApJ, 322, 374

Mathis, J. S., Rumpl, W., \& Nordsieck, K. H. 1977, ApJ, 217, 425

Meeus, G., Waters, L. B. F. L., Bouwman, J., van den Ancker, M. E., Waelkens, C., \& Malfait, K. 2001, A\&A, 365, 476

Meyer, M. R. 2001, BAAS, 198, 25.06

Miyake, K. \& Nakagawa, Y. 1993, Icarus, 106, 20

Molster, F. J., Waters, L. B. F. M., \& Tielens, A. G. G. M. 2002a, A\&A, 382,184

2002b, A\&A, 382, 222

Moro-Martín, A., \& Malhotra, R. 2002, AJ, 124, 2305 2003, AJ, 125, 2255

Noyes, R. W., Jha, S., Kozennik, S. G., Brown, T. M., Kennelly, E. J., \& Horner, S. D. 1997, ApJ, 483, L111

Pantin, E., Lagage, P. O., \& Artymowicz, P. 1997, A\&A, 327, 1123

Paresce, F., \& Burrows, Ch. 1987, ApJ, 319, L23

Rice, W. K. M., Wood, K., Armitage, P. J., Whitney, B. A., \& Bjorkman, J. E. 2003, MNRAS, 342, 79

Savage, B. D. \& Mathis, J. S. 1979, ARA\&A, 17, 73

Scarrott, S., Draper, P. W., \& Warren-Smith, R. F. 1989, MNRAS, 237, 621

Skinner, C. J., Barlow, M. J., \& Justtanont, K. 1992, MNRAS, 255, 31P

Smith, B. A., \& Terrile, R. J. 1984, Science, 226, 1421

Sopka, R. J., et al. 1985, ApJ, 294, 242

Spangler, C., Sargent, A. I., Silverstone, M. D., Becklin, E. E., \& Zuckerman, B. 2001, ApJ, 555, 932

Stapelfeldt, K. R., Krist, J. E., Menard, F., Bouvier, J., Padgett, D. L., \& Burrows, C. J. 1998, ApJ, 502, L65

Sykes, M. V. 1988, ApJ, 334, L55

Sylvester, R. J., \& Skinner, C. J. 1996, MNRAS, 283, 457

Sylvester, R. J., Skinner, C. J., Barlow, M. J., \& Mannings, V. 1996, MNRAS, 279, 915

Tanaka, H., Inaba, S., \& Nakazawa, K. 1996, Icarus, 123, 450

Thi, W. F., et al. 2001, Nature, 409, 60

Trilling, D. E., \& Brown, R. H. 1998, Nature, 395, 775

Trilling, D. E., Brown, R. H., \& Rivkin, A. S. 2000, ApJ, 529, 499

Vidal-Madjar, A., Lecavelier des Etangs, A., \& Ferlet, R. 1998, Planet. Space Sci., 46, 629

Voshchinnikov, N. V. 2003, Astroph. Space Phys. Rev., 12, 1

Warren, S. G. 1984, Appl. Opt., 23, 1206

Weidenschilling, S. J., \& Davis, D. R. 2000, BAAS, 32, 52.01

Weinberger, A. J., Becklin, E. E., \& Zuckerman, B. 2003, ApJ, 584, L33

Weingartner, J. C., \& Draine, B. T. 2001, ApJ, 548, 296

Whipple, F. L. 1976, in Interplanetary Dust and Zodiacal Light, ed. H. Elsasser \& H. Fechtig (Berlin: Springer), 403

Wilner, D. J., Holman, M. J., Kuchner, M. J., \& Ho, P. T. P. 2002, ApJ, 569, L 115

Wolf, S., Padgett, D. L., \& Stapelfeldt, K. R. 2003, ApJ, 588, 373

Wolf, S. Voshchinnikov, N. V., \& Henning, Th. 2002, A\&A, 385, 365

Wood, K., Wolff, M. J., Bjorkman, J. E., \& Whitney, B. 2002, ApJ, 564 887

Wyatt, M. C., \& Dent, W. R. F. 2002, MNRAS, 334, 589

Zuckermann, B., Forveille, T., \& Kastner, J. H. 1995, Nature, 373, 494 